Linköping Studies in Science and Technology.

Thesis No. 1700

\title{
Semigroups of Sets Without the Baire Property In Finite Dimensional Euclidean Spaces
}

\section{Vénuste NYAGAHAKWA}

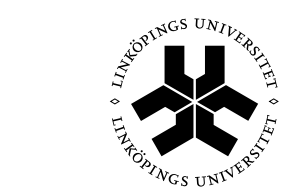

Linköpings universitet

INSTITUTE OF TECHNOLOGY

Department of Mathematics

Division of Mathematics and Applied Mathematics Linköping University, SE-581 83 Linköping, Sweden

Linköping 2015 
Linköping Studies in Science and Technology.

Thesis No. 1700

Semigroups of Sets Without the Baire Property In Finite Dimensional Euclidean Spaces

Vénuste NYAGAHAKWA

venuste.nyagahakwa@liu.se

www.mai.liu.se

Mathematics and Applied Mathematics

Department of Mathematics

Linköping University

SE-581 83 Linköping

Sweden

ISBN 978-91-7519-146-1ＩSSN 0280-7971

Copyright @ 2015 Vénuste NYAGAHAKWA

Printed by LiU-Tryck, Linköping, Sweden 2015 
To Iribagiza M. Rose

and

Mizero B. Roberto. 



\section{Abstract}

A semigroup of sets is a family of sets closed under finite unions. This thesis focuses on the search of semigroups of sets in finite dimensional Euclidean spaces $\mathbb{R}^{n}, n \geq 1$, which elements do not possess the Baire property, and on the study of their properties.

Recall that the family of sets having the Baire property in the real line $\mathbb{R}$, is a $\sigma$-algebra of sets, which includes both meager and open subsets of $\mathbb{R}$. However, there are subsets of $\mathbb{R}$ which do not belong to the algebra. For example, each classical Vitali set on $\mathbb{R}$ does not have the Baire property.

It has been shown by Chatyrko that the family of all finite unions of Vitali sets on the real line, as well as its natural extensions by the collection of meager sets, are (invariant under translations of $\mathbb{R}$ ) semigroups of sets which elements do not possess the Baire property.

Using analogues of Vitali sets, when the group $\mathbb{Q}$ of rationals in the Vitali construction is replaced by any countable dense subgroup $Q$ of reals, (we call the sets Vitali $Q$-selectors of $\mathbb{R}$ ) and Chatyrko's method, we produce semigroups of sets on $\mathbb{R}$ related to $Q$, which consist of sets without the Baire property and which are invariant under translations of $\mathbb{R}$. Furthermore, we study the relationship in the sense of inclusion between the semigroups related to different $Q$. From here, we define a supersemigroup of sets based on all Vitali selectors of $\mathbb{R}$. The defined supersemigroup also consists of sets without the Baire property and is invariant under translations of $\mathbb{R}$. Then we extend and generalize the results from the real line to the finite-dimensional Euclidean spaces $\mathbb{R}^{n}, n \geq 2$, and indicate the difference between the cases $n=1$ and $n \geq 2$. Additionally, we show how the covering dimension can be used in defining diverse semigroups of sets without the Baire property. 



\section{Populärvetenskaplig sammanfattning}

En semigrupp av mängder är en familj av mängder som är sluten under ändliga unioner. Denna licentiatavhandling fokuserar på sökandet efter semigrupper av mängder i de ändligdimensionella euklidiska rummen $\mathbb{R}^{n}, n \geq 1$, vars element inte har Baire-egenskapen, och på studiet av deras egenskaper.

Som bekant är familjen av delmängder av $\mathbb{R}$ som har Baire-egenskapen en $\sigma$ algebra av mängder som innehåller både de öppna och de magra delmängderna av $\mathbb{R}$. Det finns dock delmängder av $\mathbb{R}$ som inte tillhör algebran. Till exempel gäller att varje klassisk Vitali-mängd på $\mathbb{R}$ inte har Baire-egenskapen.

Chatyrko har visat att familjen av alla ändliga unioner av Vitali-mängder på reella linjen, och den utvidgning av denna familj som på ett naturligt sätt ges av familjen av magra mängder, är semigrupper av mängder på $\mathbb{R}$, som består av mängder som inte har Baire-egenskapen och som är invarianta under translationer av $\mathbb{R}$.

Genom att använda motsvarigheten till Vitali-mängder när gruppen $\mathbb{Q}$ i Vitalis konstruktion ersätts med en godtycklig uppräknelig tät delgrupp $Q$ av $\mathbb{R}$ (vi kallar dessa mängder Vitali- $Q$-selektorer på $\mathbb{R}$ ), och Chatyrkos metoder, konstruerar vi semigrupper av mängder på $\mathbb{R}$ relaterade till $Q$, som består av mängder som inte har Baire-egenskapen och som är invarianta under translationer av $\mathbb{R}$. Vidare studerar vi hur semigrupper relaterade till olika grupper $Q$ förhåller sig med avseende på inklusion, och vi definierar en "supersemigrupp" baserad på alla Vitali-selektorer på $\mathbb{R}$. Denna supersemigrupp består också av mängder som inte har Baire-egenskapen och är invariant under translationer av $\mathbb{R}$. Vi utvidgar och generaliserar sedan dessa resultat från reella linjen till ändligdimensionella euklidiska rum $\mathbb{R}^{n}, n \geq 2$, och visar på olikheterna i de två fallen $n=1$ och $n \geq 2$. Dessutom visar vi hur begreppet övertäckningsdimension kan användas för att definiera en mångfald av semigrupper av mängder som inte har Baireegenskapen. 



\section{Acknowledgments}

This Licentiate thesis appears in its current form due to the assistance and guidance of several people. I would therefore like to offer my sincere thanks to all of them.

First, I would like to express my gratitude to my supervisors Vitalij A. Chatyrko and Mats Aigner for useful comments, remarks and engagement through the learning process until to this Licentiate thesis. They provided encouragement and advise necessary for me to complete this Licentiate thesis and to proceed through the Ph.D program.

In a very special way, I would also like to thank Bengt Ove Turesson, Björn Textorius and all members of the Department of Mathematics for their helps whenever need arises at work. They have directed me through various situations, allowing me to reach this accomplishment. I thank Minani Froduald, Lyambabaje Alexandre and Mahara Isidore for their assistance and guidance and the helpful part they played in my mathematical developments as my teachers.

I would like to thank all my fellow Ph.D students whom I shared so many excellent times at Linköping University. In particular, I would like to thank Anna Orlof for organizing team building activities.

My family has supported and helped me along the learning process by giving encouragement and providing the moral and emotional support I needed to complete my thesis. To them, I am eternally grateful.

I wish to acknowledge the financial support I received through Sida/Sarec funded University of Rwanda-Linköping University cooperation. All involved institutions and people are hereby acknowledged.

May the Almighty God richly bless all of you.

Linköping, March 24, 2015

Vénuste NYAGAHAKWA 



\section{Contents}

1 Introduction 3

1.1 Problem formulation . . . . . . . . . . . . . . 3

1.2 Summary of main results ................. 4

1.3 Structure of the thesis ................. 6

2 Necessary facts 9

2.1 Algebraic notions in set theory . . . . . . . . . . . . 9

2.2 Some topological concepts . . . . . . . . . . . . . 11

2.2.1 Baire Category Theorem . . . . . . . . . . . . . . . 11

2.2.2 Baire property . . . . . . . . . . . . 13

2.2.3 Lebesgue covering dimension . . . . . . . . . . . . . 13

3 Algebra of semigroups of sets $\quad 15$

3.1 Semigroups and ideals of sets . . . . . . . . . . . 15

3.2 Extension of a semigroup of sets via an ideal of sets . . . . . . . 18

4 Semigroups of sets defined by Vitali selectors on the real line 23

4.1 Vitali selectors of the real line . . . . . . . . . . . . . 24

4.2 Countable dense subgroups of $\mathbb{R}$ and generated semigroups . . . . 28

4.3 Supersemigroup based on Vitali selectors of $\mathbb{R} \ldots \ldots$. . . . . . . . 31 
4.4 Semigroup of non-Lebesgue measurable sets . . . . . . . . . . . 34

5 Semigroups of sets without the Baire property in finite dimensional Euclidean spaces

5.1 Vitali selectors of $\mathbb{R}^{n} \ldots \ldots \ldots \ldots \ldots \ldots$

5.2 Supersemigroup of Vitali selectors of $\mathbb{R}^{n} \ldots \ldots$. . . . . . . . . 39

5.3 Rectangular Vitali selectors of $\mathbb{R}^{n} \ldots \ldots \ldots$. . . . . . . . . . 41

5.3.1 Supersemigroup of rectangular Vitali selectors of $\mathbb{R}^{n} \ldots$. . 46

5.4 Semigroups of sets in $\mathbb{R}^{n}$ defined by dimension . . . . . . . . . . 49

Bibliography 


\section{Notation}

The principal notation used throughout the text is listed below.

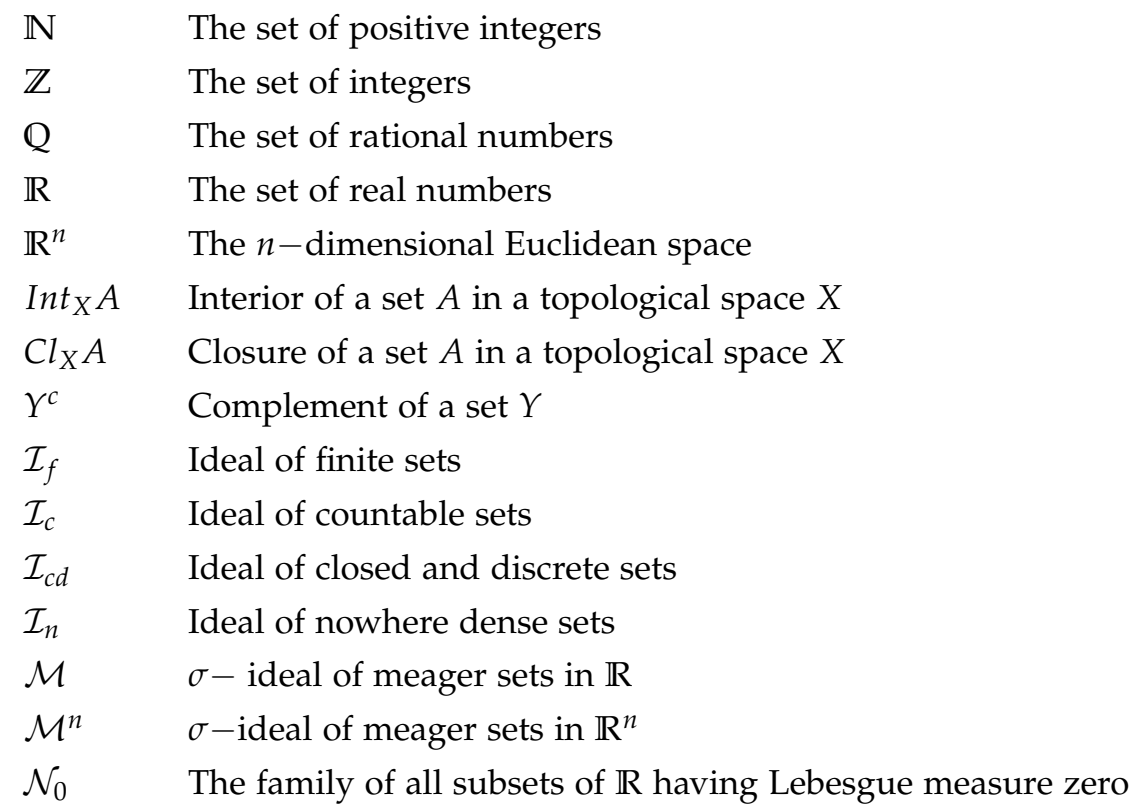


$\mathcal{N} \quad$ The family of all measurable subsets of $\mathbb{R}$ in the Lebesgue sense

$\Delta, \cup, \cap, \backslash$ Standard set operation of symmetric difference, union, intersection and set difference

dim Lebesgue covering dimension

$\mathcal{P}(X) \quad$ The family of all subsets of $X$

$\mathcal{O} \quad$ The family of all open subsets of $\mathbb{R}$

$\mathcal{O}^{n} \quad$ The family of all open subsets of $\mathbb{R}^{n}$

$\mathcal{B}_{p} \quad$ The family of sets with the Baire property on the real line

$\mathcal{B}_{p}^{n} \quad$ The family of sets with the Baire property in $\mathbb{R}^{n}$

$\mathcal{F} \quad$ The family of all countable, dense in the real line subgroups of $(\mathbb{R},+)$

$\mathcal{F}^{n} \quad$ The family of all countable, dense in the Euclidean spaces $\mathbb{R}^{n}, n \geq 2$ subgroups of $\left(\mathbb{R}^{n},+\right)$

$r \mathcal{F}^{n} \quad$ The family of all rectangular subgroups of $\left(\mathbb{R}^{n},+\right)$

$\mathcal{S}_{\mathcal{A}} \quad$ Semigroup of sets generated by $\mathcal{A}$

$\mathcal{I}_{\mathcal{A}} \quad$ Ideal of sets generated by $\mathcal{A}$

$\mathcal{V}(Q) \quad$ Family of all Vitali- $Q$ selectors of $\mathbb{R}$ associated to the subgroup $Q$ of $(\mathbb{R},+)$

$\mathcal{S}_{\mathcal{V}(Q)} \quad$ Semigroup of sets generated by $\mathcal{V}(Q)$

$\mathcal{S}_{\mathcal{V}^{\text {sup }}} \quad$ Semigroup of sets generated by $\mathcal{V}^{\text {sup }}$

$\mathcal{V}^{n}(Q) \quad$ The family of all Vitali- $Q$ selectors of $\mathbb{R}^{n}$ associated to the subgroup $Q$ of $\left(\mathbb{R}^{n},+\right)$

$\mathcal{S}_{\mathcal{V}^{n} Q} \quad$ Semigroup of sets generated by $\mathcal{V}^{n}(Q)$

$r \mathcal{V}^{n}(Q) \quad$ The family of all rectangular Vitali- $Q$ selectors of $\mathbb{R}$ associated to the subgroup $Q$ of $\left(\mathbb{R}^{n},+\right)$

$\mathcal{S}_{r \mathcal{V}^{n} Q} \quad$ Semigroup of sets generated by $r \mathcal{V}^{n}(Q)$

$\mathcal{S}_{r \mathcal{V}_{\text {sup }}^{n}} \quad$ Semigroups of sets generated by $r \mathcal{V}_{\text {sup }}^{n}$ 


\section{Introduction}

\subsection{Problem formulation}

Let $\mathbb{R}$ be the set of real numbers and $\mathcal{P}(\mathbb{R})$ the family of all subsets of $\mathbb{R}$. Furthermore, let $\left(\mathbb{R}, \tau_{E}\right)$ be the real line, i.e. the set $\mathbb{R}$ endowed with the topology $\tau_{E}$ defined by all open intervals of $\mathbb{R}$, and $\mathcal{M}$ the family of all meager subsets of $\left(\mathbb{R}, \tau_{E}\right)$.

An interesting extension of $\mathcal{M}$, as well as $\tau_{E}$ in $\mathcal{P}(\mathbb{R})$, is the family $\mathcal{B}_{p}$ of all subsets of $\left(\mathbb{R}, \tau_{E}\right)$ possessing the Baire property. Recall ([1]) that a set $B \in \mathcal{B}_{p}$ if and only if there are an $O \in \tau_{E}$ and an $M \in \mathcal{M}$ such that $B=O \Delta M$.

It is well known (see [1]) that $\mathcal{B}_{p} \neq \mathcal{P}(\mathbb{R})$ (for example, each Vitali set $V$ of $\mathbb{R}[2]$ is an element of the complement $\mathcal{B}_{p}^{c}=\mathcal{P}(\mathbb{R}) \backslash \mathcal{B}_{p}$ of $\mathcal{B}_{p}$ in $\mathcal{P}(\mathbb{R})$ ), and the family $\mathcal{B}_{p}$ is a $\sigma$-algebra of sets, in particular, $\mathcal{B}_{p}$ is closed under finite unions and finite intersections of sets. Let us also note that the family $\mathcal{B}_{p}$ is invariant under action of the group $\mathcal{H}\left(\left(\mathbb{R}, \tau_{E}\right)\right)$ of all homeomorphisms of the real line $\left(\mathbb{R}, \tau_{E}\right)$ onto itself, i.e. for each $B \in \mathcal{B}_{p}$ and each $h \in \mathcal{H}\left(\left(\mathbb{R}, \tau_{E}\right)\right)$ we have $h(B) \in \mathcal{B}_{p}$.

It is easy to see that the family $\mathcal{B}_{p}^{c}$ is also invariant under action of the group $\mathcal{H}\left(\left(\mathbb{R}, \tau_{E}\right)\right)$ but, unlike the family $\mathcal{B}_{p}, \mathcal{B}_{p}^{c}$ is not closed under finite unions and finite intersections of sets. It is also well known (cf. [3]) that there are elements of $\mathcal{B}_{p}^{c}$ with a natural algebraic structure (for example, some subgroups of the 
additive group $(\mathbb{R},+)$ of all real numbers).

One can pose the following problem ([4]):

Do there exist subfamilies of $\mathcal{B}_{p}^{c}$ which are invariant under action of an infinite subgroup of $\mathcal{H}\left(\left(\mathbb{R}, \tau_{E}\right)\right)$ and on which we can define some algebraic structure?

The following simple observation can give an answer to the question.

Let $\mathcal{G}$ be a non-trivial sufficiently rich subgroup $\mathcal{G}$ of $\mathcal{H}\left(\left(\mathbb{R}, \tau_{E}\right)\right)$ and $\mathcal{A}$ be a subfamily of $\mathcal{B}_{p}^{c}$ such that $\mathcal{A}$ is invariant under action of $\mathcal{G}$. If for each $n \geq 2$ and each $A_{1}, A_{2}, \cdots, A_{n} \in \mathcal{A}$ we have $A_{1} \cup A_{2} \cup \cdots A_{n} \in \mathcal{B}_{p}^{c}$ then the family $\mathcal{S}_{\mathcal{A}}$ consisting of all finite unions of elements of $\mathcal{A}$ is a semigroup of sets with respect to the binary operation " $\cup$ " union of sets, which is invariant under action of $\mathcal{G}$ and which is in $\mathcal{B}_{p}^{c}$.

In [5], Chatyrko proved that any union of finitely many Vitali sets is an element of $\mathcal{B}_{p}^{c}$. It is easy to see that the family $\mathcal{V}$ of all Vitali sets is invariant under action of the group $\tau(\mathbb{R})$ of all translations of $\mathbb{R}$. Hence, by the observation above, the family $\mathcal{S}_{\mathcal{V}}$ of all finite unions of Vitali sets is a semigroup of sets with respect to the operation " $\cup$ ", which is invariant under action of $\tau(\mathbb{R})$ and which is in $\mathcal{B}_{p}^{c}$. Furthermore, in [4] Chatyrko proved that the family $\mathcal{S}_{\mathcal{V}} \Delta \mathcal{M}=\left\{U \Delta M: U \in \mathcal{S}_{\mathcal{V}}, M \in \mathcal{M}\right\}$ is also a semigroup of sets with the respect to the operation " $\cup$ ", which is invariant under action of $\tau(\mathbb{R})$ and which is in $\mathcal{B}_{p}^{c}$.

The goals of this thesis are the following.

(a) In the realm of $\mathcal{P}(\mathbb{R})$ to find families of sets (different from the families $\mathcal{S}_{\mathcal{V}}$ and $\mathcal{S}_{\mathcal{V}} \Delta \mathcal{M}$ mentioned above) which are semigroups of sets with respect to the operation " $\cup$ ", which are invariant under action of $\tau(\mathbb{R})$ and which are in $\mathcal{B}_{p}^{c}$.

(b) To extend the results of (a) to Euclidean spaces $\mathbb{R}^{n}, n \geq 2$.

\subsection{Summary of main results}

Let $X$ be a non-empty set and let $\mathcal{P}(X)$ be the family of all subsets of $X$.

For families of sets $\mathcal{A}$ and $\mathcal{B}$ in $\mathcal{P}(X)$, we define two operations:

$\mathcal{A} \cup \mathcal{B}=\{A \cup B: A \in \mathcal{A}, B \in \mathcal{B}\}$,

$\mathcal{A} \Delta \mathcal{B}=\{A \Delta B: A \in \mathcal{A}, B \in \mathcal{B}\}$.

However, by $\mathcal{A} \cap \mathcal{B}$ we denote the family of common elements of $\mathcal{A}$ and $\mathcal{B}$. 
Moreover, if $\mathcal{D} \subset \mathcal{P}(X)$ then by $\mathcal{S}_{\mathcal{D}}$ we mean the family of all finite unions of elements of $\mathcal{D}$.

In Chapter 3, we have obtained the following results (Proposition 3.2 and Proposition 3.4):

(a) If $\mathcal{S}$ is a semigroup of sets with respect to the operation " $\cup$ " and $\mathcal{I}$ is an ideal of sets in $\mathcal{P}(X)$, then the families $\mathcal{S} \cup \mathcal{I}, \mathcal{S} \Delta \mathcal{I}$ are semigroups of sets with respect to the operation " $\cup$ " and $\mathcal{S} \subset \mathcal{S} \cup \mathcal{I} \subset \mathcal{S} \Delta \mathcal{I}$.

(b) Let $\mathcal{I}$ be an ideal of sets and $\mathcal{A}, \mathcal{B} \subset \mathcal{P}(X)$ such that $\mathcal{A} \cap \mathcal{I}=\varnothing$ and for each element $U \in \mathcal{S}_{\mathcal{A}}$ and each non-empty element $B \in \mathcal{B}$ there is an element $A \in \mathcal{A}$ satisfying $A \subset B \backslash U$. Then $\left(S_{\mathcal{A}} \Delta \mathcal{I}\right) \cap\left(\mathcal{S}_{\mathcal{B}} \Delta \mathcal{I}\right)=\varnothing$.

In Chapter 4, the main attention was paid to the case when $X=\mathbb{R}$.

Let $Q$ be a countable, dense in the real line, subgroup of $(\mathbb{R},+), \mathcal{V}(Q)$ be the family of all Vitali $Q$-selectors of $\mathbb{R}$ associated to $Q$ (analogues of Vitali sets, considered in [3], when the set $\mathbb{Q}$ of rationals is substituted by $Q$ ) and $\mathcal{I}$ any subideal of $\mathcal{M}$. Using the Chatyrko's method, the results from the previous chapter and the observation that $\tau_{E}=\mathcal{S}_{\tau_{E}}$, we have proved (Proposition 4.3) that:

(c) The families $\mathcal{S}_{\mathcal{V}(Q)}, \mathcal{S}_{\mathcal{V}(Q)} \cup \mathcal{I}$ and $\mathcal{S}_{\mathcal{V}(Q)} \Delta \mathcal{I}$ are semigroups of sets with respect to the operation " $\cup$ " and $\mathcal{S}_{\mathcal{V}(Q)} \subset \mathcal{S}_{\mathcal{V}(Q)} \cup \mathcal{I} \subset \mathcal{S}_{\mathcal{V}(Q)} \Delta \mathcal{I}$. Moreover, $\mathcal{S}_{\mathcal{V}(Q)}, \mathcal{S}_{\mathcal{V}(Q)} \cup \mathcal{I}$ and $\mathcal{S}_{\mathcal{V}(Q)} \Delta \mathcal{I}$ are invariant under action of $\tau(\mathbb{R})$, and consist of sets without the Baire property.

We have also observed that in the family $\left\{\mathcal{S}_{\mathcal{V}(Q)}: Q \subset \mathbb{R}\right\}$ there is no element which contains all others (Proposition 4.8). So we consider the family $\mathcal{V}^{\text {sup }}$ of all Vitali $Q$-selectors of $\mathbb{R}$, where $Q$ is varied, and the semigroup $\mathcal{S}_{\mathcal{V}}$ sup which we call a supersemigroup of Vitali selectors. The supersemigroup $\mathcal{S}_{\mathcal{V}}$ sup contains the semigroup $\mathcal{S}_{\mathcal{V}(Q)}$ for each $Q$.

In the same way as above we have proved (Theorem 4.1) that:

(d) The families $\mathcal{S}_{\mathcal{V} \text { sup , }} \mathcal{S}_{\mathcal{V} \text { sup }} \cup \mathcal{I}$ and $\mathcal{S}_{\mathcal{V} \text { sup }} \Delta \mathcal{I}$ are semigroups of sets with respect to the operation " $\cup$ " and $\mathcal{S}_{\mathcal{V} \text { sup }} \subset \mathcal{S}_{\mathcal{V} \text { sup }} \cup \mathcal{I} \subset \mathcal{S}_{\mathcal{V} \text { sup }} \Delta \mathcal{I}$. Moreover, $\mathcal{S}_{\mathcal{V} \text { sup }}, \mathcal{S}_{\mathcal{V} \text { sup }} \cup$ $\mathcal{I}$ and $\mathcal{S}_{\mathcal{V} \text { sup }} \Delta \mathcal{I}$ are invariant under action of $\tau(\mathbb{R})$, and consist of sets without the Baire property. 
In Chapter 5, the main attention was paid to the case when $X=\mathbb{R}^{n}, n \geq 2$. Let $Q$ be a countable, dense in the Euclidean space $\mathbb{R}^{n}$, subgroup of $\left(\mathbb{R}^{n},+\right)$, $\mathcal{V}^{n}(Q)$ be the family of all Vitali $Q$-selectors of $\mathbb{R}^{n}$ associated to $Q$ (analogues of Vitali $Q$-selectors for the real line, see also [3]) and $\mathcal{I}$ any subideal of the family $\mathcal{M}^{n}$ of all meager sets of $\mathbb{R}^{n}$. Using the results and technique from the previous chapters we have proved statements which are similar to (c) and (d).

Moreover, we have pointed out a special case of Vitali $Q$-selectors of $\mathbb{R}^{n}$ called rectangular Vitali selectors of $\mathbb{R}^{n}, n \geq 2$, related to groups $Q$ which are products of $n$ many countable dense in the real line groups. Our rectangular Vitali selectors are supposed to be products of $n$ many Vitali selectors of the real line. We have extended our theory to the special case. Since the rectangular products are somewhat less complicated than general ones, we could obtain more information about them.

A part of the mentioned results can be found in [4] and [6].

\subsection{Structure of the thesis}

This thesis contains five chapters which are structured as follows:

The first chapter gives a brief description of the problem under investigation and a brief summary of the obtained results.

The second chapter introduces basic concepts, terminology and statements which will be needed for a better understanding of the subsequent chapters.

The third chapter treats the theory of semigroups of sets with respect to the operation of union of sets. Through various examples, we describe the behaviour of semigroups of sets with the respect to several binary operations. Furthermore, we present a way of searching pairs of semigroups of sets without common elements.

The fourth chapter develops the theory of semigroups of sets without the Baire property on the real line. These semigroups are constructed by using Vitali $Q$-selectors of $\mathbb{R}$ and subideals of the ideal of meager subsets of $\mathbb{R}$. They are invariant under translations of the real line and they consist of sets without the Baire property.

The last fifth chapter generalizes and extends the results of Chapter 4 to 
the finite-dimensional Euclidean spaces $\mathbb{R}^{n}, n \geq 2$. Besides the semigroups of sets generated by ordinary Vitali selectors, the chapter treats also semigroups of sets generated by rectangular Vitali selectors of $\mathbb{R}^{n}, n \geq 2$. Rectangular Vitali selectors are a special case of the ordinary ones. In both cases, the generated semigroups of sets are invariant under translations of $\mathbb{R}^{n}$ and they consist of sets without the Baire property. The chapter ends by pointing out the role of dimension in defining different semigroups of sets without the Baire property. 



\section{Necessary facts}

The purpose of this chapter is to recall concepts and terminology which will be used in the subsequent chapters.

\subsection{Algebraic notions in set theory}

In this section, we shall give a short introduction to families of sets with algebraic properties. By a family of sets, we mean any set whose elements are themselves sets. Families of sets are denoted by capital script letters like $\mathcal{S}, \mathcal{O}$ and so forth.

For a more detailed information, we refer the reader to one of the references [7], [8] or [9].

Let $X$ be a non-empty set and let $\mathcal{P}(X)$ be the family of all subsets of $X$.

Definition 2.1. A non-empty family $\mathcal{R} \subset \mathcal{P}(X)$ of sets is called a ring of sets on $X$ if $A \Delta B \in \mathcal{R}$ and $A \cap B \in \mathcal{R}$ whenever $A \in \mathcal{R}, B \in \mathcal{R}$.

Since $A \cup B=(A \Delta B) \Delta(A \cap B), A \backslash B=A \Delta(A \cap B)$, we have also $A \cup B \in \mathcal{R}$ and $A \backslash B \in \mathcal{R}$ whenever $A \in \mathcal{R}, B \in \mathcal{R}$. Thus a ring is a family of sets closed under the operations of taking unions, intersections, differences and symmetric differences. A ring of sets must contain the empty set $\varnothing$, since $A \backslash A=\varnothing$.

Definition 2.2. Let $\mathcal{A} \subseteq \mathcal{P}(X)$ be a ring of sets on $X$. If $X \in \mathcal{A}$, the family $\mathcal{A}$ is called an algebra of sets on $X$. 
From this definition, a ring of sets is an algebra if and only if it closed under taking the operation of complement.

Example 2.1

(i) The family of all finite subsets of $X$ is a ring on $X$ but not an algebra on $X$ unless $X$ is finite.

(ii) Let $\mathbb{R}$ be the set of real numbers. The family of all bounded subsets of $\mathbb{R}$ is a ring on $\mathbb{R}$ but not an algebra.

Definition 2.3. (a) A ring $\mathcal{R} \subset \mathcal{P}(X)$ is called a $\sigma$-ring of sets on $X$ if it is closed under countable unions, i.e. it contains the union $S=\bigcup_{n=1}^{\infty} A_{n}$ whenever it contains the sets $A_{1}, A_{2}, \ldots$

(b) A $\sigma$-ring $\mathcal{A} \subset \mathcal{P}(X)$ is called a $\sigma$-algebra of sets on $X$ if $X \in \mathcal{A}$.

From the De Morgan formula $\bigcap_{n=1}^{\infty} A_{n}=X \backslash \bigcup_{n=1}^{\infty}\left(X \backslash A_{n}\right)$, it follows that each $\sigma$-algebra is also closed under countable intersection of sets.

Note that that a $\sigma$-algebra can be defined as an algebra closed under countable unions.

\section{Example 2.2}

For a set $X$, the family of all countable subsets of $X$ is a $\sigma$-ring. It will be a $\sigma$-algebra if $X$ is countable.

Definition 2.4. Let $\mathcal{A} \subset \mathcal{P}(X)$. The smallest $\sigma$ - algebra of sets on $X$ containing $\mathcal{A}$ is called a $\sigma$-algebra generated by the family $\mathcal{A}$.

\section{Example 2.3}

Let $\mathbb{R}$ be the real line, i.e. the set $\mathbb{R}$ endowed with the standard metric $\rho$ defined by $\rho(x, y)=|x-y|$ for all $x, y \in \mathbb{R}$ and $\mathcal{N}$ be the family of all measurable subsets of $\mathbb{R}$ in the Lebesgue sense. The family $\mathcal{N}$ is a $\sigma$-algebra of sets which is generated by $\mathcal{N}_{0} \cup \mathcal{O}$, where $\mathcal{N}_{0}$ is the family of all subsets of $\mathbb{R}$ having Lebesgue measure zero and $\mathcal{O}$ is the family of all open subsets of $\mathbb{R}$. 
Definition 2.5. (a) A family $\mathcal{I} \subset \mathcal{P}(X)$ of sets is called an ideal of sets on $X$, if it satisfies the following two conditions:

(i) If $A \in \mathcal{I}$ and $B \in \mathcal{I}$ then $A \cup B \in \mathcal{I}$.

(ii) If $A \in \mathcal{I}$ and $B \subset A$ then $B \in \mathcal{I}$.

(b) If an ideal of sets $\mathcal{I}$ is closed under countable unions, then it is called a $\sigma$-ideal of sets on $X$.

\section{Example 2.4}

Let $A \subseteq X$. Then

(i) the family $\mathcal{I}(A)=\{B: B \subseteq A\}$ is a $\sigma$-ideal of sets on $X$,

(ii) the family $\mathcal{I}_{c}$ of all countable subsets of $X$ forms a $\sigma$-ideal of sets on $X$,

(iii) the family $\mathcal{I}_{f}$ of all finite subsets of $X$ forms an ideal of sets on $X$, but not a $\sigma$-ideal of sets, whenever $X$ is infinite.

\subsection{Some topological concepts}

\subsubsection{Baire Category Theorem}

Let $X$ be a topological space and let $A$ be a subset of $X$.

Recall that a neighborhood of a point $x \in X$ is any open subset $U$ of $X$ containing $x$. The point $x \in X$ is a limit point of $A$ if $(U \backslash\{x\}) \cap A \neq \varnothing$ for every neighborhood $U$ of $x$. The derived set of $A$, denoted by $A^{d}$, is the set of all limit points of $A$.

Definition 2.6. A subset $A$ of $X$ is said to be closed and discrete if and only if $A^{d}=\varnothing$.

Note that each subset of a closed and discrete subset of $X$ (resp. each finite union of closed discrete subsets of $X$ ) is also a closed discrete subset of $X$. Thus the family of all closed and discrete subsets of $X$ forms an ideal of sets, denoted by $\mathcal{I}_{c d}$. 
Definition 2.7. A subset $A \subset X$ is called a nowhere dense set in $X$ if $\operatorname{Int}_{X}\left(C l_{X}(A)\right)=\varnothing$.

It is easy to see that every subset of a nowhere dense set is a nowhere dense set, and the union of finitely many nowhere dense sets is again a nowhere dense set. Thus, the family of nowhere dense sets in a given topological space forms an ideal of sets, denoted by $\mathcal{I}_{n}$.

Example 2.5

Every finite subset of the real line $\mathbb{R}$, the set $\mathbb{Z}$ of all integers and the Cantor set, are nowhere dense subsets of $\mathbb{R}$.

Definition 2.8. A subset $A \subset X$ is said to be dense in $X$ if $C l_{X}(A)=X$.

Example 2.6

(1) The set $\mathbb{Q}$ of all rational numbers is a dense subset of $\mathbb{R}$.

(2) The set $\mathbb{Z}(\sqrt{2})=\{n+\sqrt{2} m: n \in \mathbb{Z}, m \in \mathbb{Z}\}$ is a dense subset of $\mathbb{R}[10]$.

Remark 2.1. Note that a countable union of nowhere dense sets is not necessarily a nowhere dense set. For example, the set $\mathbb{Q}$ of rationals is a union of countably many nowhere dense sets in $\mathbb{R}$, but $\operatorname{Int}_{\mathbb{R}}\left(C l_{\mathbb{R}}(\mathbb{Q})\right)=\mathbb{R}$.

Definition 2.9. A subset $A \subset X$ is meager (or of first category) if $A$ is the union of countably many nowhere dense sets. Any set that is not meager is said to be nonmeager (or of second category).

Example 2.7

The set $\mathbb{Q}$ of rationals numbers is a meager subset of the real line $\mathbb{R}$.

In a given topological space, the family of all meager sets forms a $\sigma$-ideal of sets. The $\sigma$-ideal of meager sets will be denoted by $\mathcal{M}$ in our further considerations.

A fundamental theorem of Baire asserts the following [11], [12]. 
Theorem 2.1 (Baire Category Theorem). Let X be a complete metric space. Then X can not be covered by countably many nowhere dense subsets. Moreover, the union of countably many nowhere dense subsets of $X$ has a dense complement.

Recall that the real line $\mathbb{R}$ is a complete metric space. So by Baire Category Theorem, $\mathbb{R}$ is of the second category.

Similarly, the Euclidean space $\mathbb{R}^{n}, n \geq 1$, i.e. the set $\mathbb{R}^{n}$ endowed with the metric $\rho(x, y)=\sqrt{\sum_{i=1}^{n}\left(x_{i}-y_{i}\right)^{2}}$, where $x=\left(x_{1}, \cdots, x_{n}\right)$ and $y=\left(y_{1}, \cdots, y_{n}\right)$, is a complete metric space. So $\mathbb{R}^{n}$ is of the second category.

Remark 2.2. On the real line $\mathbb{R}$ the ideals of sets $\mathcal{I}_{f}, \mathcal{I}_{c d}, \mathcal{I}_{c}, \mathcal{I}_{n}$ and $\mathcal{M}$ satisfy the inclusions $\mathcal{I}_{f} \subsetneq \mathcal{I}_{c d} \subsetneq \mathcal{I}_{c} \subsetneq \mathcal{M}$ and $\mathcal{I}_{f} \subsetneq \mathcal{I}_{c d} \subsetneq \mathcal{I}_{n} \subsetneq \mathcal{M}$.

Note that $\mathcal{I}_{c}$ and $\mathcal{I}_{n}$ are not comparable in the sense of inclusion on $\mathbb{R}$. In fact, the Cantor set is uncountable and nowhere dense subset of $\mathbb{R}$ while the set $\mathbb{Q}$ of rationals numbers is a countable dense subset of $\mathbb{R}$.

\subsubsection{Baire property}

In this subsection, $X$ is assumed to be a topological space.

Definition 2.10. A subset $A \subset X$ is said to have the Baire property if it can be represented in the form $A=O \Delta M$, where $O$ is an open set of $X$ and $M$ is a meager set of $X$. (Recall that $O \Delta M=(O \backslash M) \cup(M \backslash O)$ )

Note that a subset $A \subset X$ has the Baire property in $X$ if and only if there is an open set $O$ of $X$ and two meager sets $M, N$ of $X$ such that $A=(O \backslash M) \cup N$.

The family of all sets with the Baire property in a topological space $X$ will be denoted by $\mathcal{B}_{p}$ in our further considerations.

Recall that the family $\mathcal{B}_{p}$ is a $\sigma$-algebra of sets. In particular, each open set of $X$ and each meager set of $X$ have the Baire property. Thus, the $\sigma$-algebra $\mathcal{B}_{p}$ is the smallest $\sigma$-algebra containing all open and all meager sets in $X$.

\subsubsection{Lebesgue covering dimension}

In this subsection, we present some basic properties of Lebesgue covering dimension dim.

Let $X$ be a topological space and let $\mathcal{A}=\left\{A_{\alpha}\right\}_{\alpha \in \Gamma}$ be a family of subsets of $X$, where $\Gamma$ is an index set. 
Definition 2.11. (i) The order of the family $\mathcal{A}=\left\{A_{\alpha}\right\}_{\alpha \in \Gamma}$ of subsets, not all empty, of $X$, is the largest integer $n$ for which there exists a subset $I$ of $\Gamma$ with $n+1$ elements such that $\cap_{\alpha \in I} A_{\alpha}$ is non-empty, or $\infty$ if there is no such largest integer.

(ii) The family $\mathcal{A}=\left\{A_{\alpha}\right\}_{\alpha \in \Gamma}$ is a cover of $X$ if $\bigcup_{\alpha \in \Gamma} A_{\alpha}=X$.

(iii) A cover $\mathcal{B}$ is a refinement of another cover $\mathcal{A}$ of the same space $X$, if for every $B \in \mathcal{B}$ there exists $A \in \mathcal{A}$ such that $B \subset A$.

Definition 2.12. Let $X$ be a topological space. Then

$\boldsymbol{\nabla} \operatorname{dim} X=-1$ if and only if $X=\varnothing$.

$\boldsymbol{\nabla} \operatorname{dim} X \leq n$ if each finite open cover of $X$ has an open refinement of order not exceeding $n$.

$\boldsymbol{\nabla} \operatorname{dim} X=n$ if it is true that $\operatorname{dim} X \leq n$ but it is not true that $\operatorname{dim} X \leq n-1$.

$\boldsymbol{\nabla} \operatorname{dim} X=\infty$ if for every integer $n$ it is false that $\operatorname{dim} X \leq n$.

If $\operatorname{dim} X=n$, then $X$ is called the $n$-dimensional topological space.

Recall that a space $X$ is said to be separable if it contains a countable dense subset. It is said to be metrizable if there exists a metric on $X$ which induces the topology on $X$.

For separable metrizable spaces, some basic properties about Lebesgue covering dimension are summarized in the following theorems [13].

Theorem 2.2 (Fundamental Theorem of Dimension). For every natural number $n$, we have $\operatorname{dim} \mathbb{R}^{n}=n$.

Theorem 2.3 (Monotonicity). If $A$ is a subspace of a separable metrizable space $X$, then $\operatorname{dim} A \leq \operatorname{dim} X$.

Theorem 2.4 (Countable Sum Theorem). Let $X$ be a separable metrizable space and $X=\bigcup_{i=1}^{\infty} F_{i}$ where $F_{i}$ is closed in $X$ for each $i$. If $\operatorname{dim} F_{i} \leq n$ for each $i$, then $\operatorname{dim} X \leq n$.

Theorem 2.5 (Product Theorem). Let $X$ and $Y$ be non-empty separable metrizable spaces. Then $\operatorname{dim}(X \times Y) \leq \operatorname{dim} X+\operatorname{dim} Y$.

Theorem 2.6 (Brouwer Dimension Theorem). Let $X \subseteq \mathbb{R}^{n}$. Then $\operatorname{dim} X=n$ if and only if $\operatorname{Int}_{\mathbb{R}^{n}}(X) \neq \varnothing$. 


\section{Algebra of semigroups of sets}

In this chapter we introduce the notion of a semigroup of sets. Then we look at the behaviour of semigroups of sets under some binary operations. Additionally, we present a way to extend a given semigroup of sets to another one by the use of ideals of sets. Before ending the chapter, we state and prove a proposition which will be used in searching of pairs of semigroups of sets without common elements. The results of this chapter were taken from the article [6].

Below $X$ is assumed to be a non-empty set and $\mathcal{P}(X)$ is the family of all subsets of $X$.

\subsection{Semigroups and ideals of sets}

Families of sets, like rings of sets or algebra of sets, are of fundamental importance in Topology and Analysis, and their properties are well known (see [9], [14]). In this section, we will consider another families of sets, namely, semigroups of sets and ideal of sets, and prove some statements about them.

Definition 3.1. A non-empty set $\mathcal{S}$ is called a semigroup if there is a binary operation $\star: \mathcal{S} \times \mathcal{S} \longrightarrow \mathcal{S}$ for which the associativity law is satisfied, i.e. the equality $(x \star y) \star z=x \star(y \star z)$ holds for all $x, y, z \in \mathcal{S}$. The semigroup $\mathcal{S}$ is called abelian if $x \star y=y \star x$ for all $x, y \in \mathcal{S}$. 
Consider a family of sets $\mathcal{S} \subseteq \mathcal{P}(X)$ such that for each pair of elements $A, B \in \mathcal{S}$ we have $A \cup B \in \mathcal{S}$. Since the union of sets is both commutative and associative, such a family of sets will be an abelian semigroup with the respect to the operation of union of sets. This observation leads to the following definition.

Definition 3.2. A non-empty family of sets $\mathcal{S} \subseteq \mathcal{P}(X)$ is called a semigroup of sets on $X$ if it is closed under finite unions.

Remark 3.1. Using the definition of a semigroup of sets, we can redefine the notion of an ideal of sets in the following way: a non-empty family $\mathcal{I} \subseteq \mathcal{P}(X)$ is an ideal of sets on $X$ iff it is a semigroup of sets on $X$ and if $A \in \mathcal{I}$ and $B \subseteq A$ then $B \in \mathcal{I}$.

Let $\mathcal{A} \subset \mathcal{P}(X)$. Put $\mathcal{S}_{\mathcal{A}}=\left\{\bigcup_{i=1}^{n} A_{i}: A_{i} \in \mathcal{A}, n \in \mathbb{N}\right\}$ and $\mathcal{I}_{\mathcal{A}}=\{B \in \mathcal{P}(X)$ : there is $A \in \mathcal{S}_{\mathcal{A}}$ such that $\left.B \subseteq A\right\}$.

The following proposition is evident.

Proposition 3.1. The family $\mathcal{S}_{\mathcal{A}}$ is a semigroup of sets on $X$ and the family $\mathcal{I}_{\mathcal{A}}$ is an ideal of sets on $X$.

We will call $\mathcal{S}_{\mathcal{A}}$ the semigroup of sets generated by the family $\mathcal{A}$ and $\mathcal{I}_{\mathcal{A}}$ will be called the ideal of sets generated by the family $\mathcal{A}$.

Let us define three binary operations on subfamilies of $\mathcal{P}(X)$ as follows.

If $\mathcal{A}, \mathcal{B} \subset \mathcal{P}(X)$ then

(1) $\mathcal{A} \cup \mathcal{B}=\{A \cup B: A \in \mathcal{A}, B \in \mathcal{B}\}$;

(2) $\mathcal{A} \Delta \mathcal{B}=\{A \Delta B: A \in \mathcal{A}, B \in \mathcal{B}\}$;

(3) $\mathcal{A} * \mathcal{B}=\left\{\left(A \backslash B_{1}\right) \cup B_{2}: A \in \mathcal{A} ; B_{1}, B_{2} \in \mathcal{B}\right\}$

where $\cup, \Delta$ and $\backslash$ are the usual union, symmetric difference of sets and difference of sets, respectively.

For the defined operations, we observe the following:

(i) Since the union and the symmetric difference of sets are commutative operations, we have $\mathcal{A} \cup \mathcal{B}=\mathcal{B} \cup \mathcal{A}$ and $\mathcal{A} \Delta \mathcal{B}=\mathcal{B} \Delta \mathcal{A}$. From the fact that $A \cup B=(A \backslash B) \cup B=(B \backslash A) \cup A$, we have $\mathcal{A} \cup \mathcal{B} \subset \mathcal{A} * \mathcal{B}$ and $\mathcal{A} \cup \mathcal{B} \subset \mathcal{B} * \mathcal{A}$. We note that if $\mathcal{A}$ and $\mathcal{B}$ are both semigroups of sets or ideals of sets, then the family $\mathcal{A} \cup \mathcal{B}$ is of the same type. 
(ii) As we will see in the following examples, in general for given semigroups of sets $\mathcal{A}$ and $\mathcal{B}$, the families $\mathcal{A} \Delta \mathcal{B}, \mathcal{A} * \mathcal{B}, \mathcal{B} * \mathcal{A}$ do not need to bemigroups of sets and none of the inclusions $\mathcal{A} \Delta \mathcal{B} \subseteq \mathcal{A} \cup \mathcal{B}, \mathcal{A} \Delta \mathcal{B} \supseteq \mathcal{A} \cup \mathcal{B}$, $\mathcal{A} \Delta \mathcal{B} \subseteq \mathcal{A} * \mathcal{B}, \mathcal{A} \Delta \mathcal{B} \supseteq \mathcal{A} * \mathcal{B}, \mathcal{A} * \mathcal{B} \subseteq \mathcal{B} * \mathcal{A}$ needs to hold. Moreover, one of the families $\mathcal{A} * \mathcal{B}, \mathcal{B} * \mathcal{A}$ can be a semigroup of sets while the other is not.

\section{Example 3.1}

Let $|X| \geq 2$ and $A$ be a non empty proper subset of $X$. Put $B=X \backslash A$, $\mathcal{A}=\{A, X\}$ and $\mathcal{B}=\{B, X\}$. Note that $\mathcal{A}=\mathcal{S}_{\mathcal{A}}, \mathcal{B}=\mathcal{S}_{\mathcal{B}}$ and the families $\mathcal{A} \cup \mathcal{B}=\{X\}, \mathcal{A} \Delta \mathcal{B}=\{\varnothing, A, B, X\}, \mathcal{A} * \mathcal{B}=\{B, X\}, \mathcal{B} * \mathcal{A}=\{A, X\}$ are semigroups of sets. Moreover, none of the following inclusions $\mathcal{A} \Delta \mathcal{B} \subseteq \mathcal{A} \cup \mathcal{B}$, $\mathcal{A} \Delta \mathcal{B} \subseteq \mathcal{A} * \mathcal{B}, \mathcal{A} * \mathcal{B} \subseteq \mathcal{B} * \mathcal{A}$ and $\mathcal{B} * \mathcal{A} \subseteq \mathcal{A} * \mathcal{B}$ holds

In our further considerations, the notation $Y^{c}$ means the complement of a set $Y$ in the set $X$.

\section{Example 3.2}

Let $X=\{1,2,3,4\}, A_{1}=\{1,3\}, A_{2}=\{2,4\}, B_{1}=\{1,2\}, B_{2}=\{3,4\}$,

$C=\{1,4\}, D=\{2,3\}, \mathcal{A}=\left\{\varnothing, A_{1}, A_{2}\right\}$ and $\mathcal{B}=\left\{\varnothing, B_{1}, B_{2}\right\}$. Note that $\mathcal{S}_{\mathcal{A}}=\left\{\varnothing, A_{1}, A_{2}, X\right\}$ and $\mathcal{S}_{\mathcal{B}}=\left\{\varnothing, B_{1}, B_{2}, X\right\}$. Moreover, we have $\mathcal{S}_{\mathcal{A}} \cup \mathcal{S}_{\mathcal{B}}=$ $\left\{\varnothing, A_{1}, A_{2}, B_{1}, B_{2},\{1\}^{c},\{2\}^{c},\{3\}^{c},\{4\}^{c}, X\right\}, \mathcal{S}_{\mathcal{A}} \Delta \mathcal{S}_{\mathcal{B}}=\left\{\varnothing, A_{1}, A_{2}, B_{1}, B_{2}, C, D, X\right\}$ and $\mathcal{S}_{\mathcal{A}} * \mathcal{S}_{\mathcal{B}}=\mathcal{S}_{\mathcal{B}} * \mathcal{S}_{\mathcal{A}}=\mathcal{P}(X) \backslash\{C, D\}$. It is easy to see that the inclusions $\mathcal{S}_{\mathcal{A}} * \mathcal{S}_{\mathcal{B}} \subseteq \mathcal{S}_{\mathcal{A}} \Delta \mathcal{S}_{\mathcal{B}}$ and $\mathcal{S}_{\mathcal{A}} \cup \mathcal{S}_{\mathcal{B}} \subseteq \mathcal{S}_{\mathcal{A}} \Delta \mathcal{S}_{\mathcal{B}}$ do not hold. We note also that none of the families $\mathcal{S}_{\mathcal{A}} \Delta \mathcal{S}_{\mathcal{B}}, \mathcal{S}_{\mathcal{A}} * \mathcal{S}_{\mathcal{B}}$ and $\mathcal{S}_{\mathcal{B}} * \mathcal{S}_{\mathcal{A}}$ are semigroups of sets. In fact, $A_{1}, D \in \mathcal{S}_{\mathcal{A}} \Delta \mathcal{S}_{\mathcal{B}}$ but $A_{1} \cup D=\{4\}^{c} \notin \mathcal{S}_{\mathcal{A}} \Delta \mathcal{S}_{\mathcal{B}}$, and $\{1\},\{4\} \in \mathcal{S}_{\mathcal{A}} * \mathcal{S}_{\mathcal{B}}$ but $\{1\} \cup\{4\}=C \notin \mathcal{S}_{\mathcal{A}} * \mathcal{S}_{\mathcal{B}}$.

\section{Example 3.3}

Let $X=\{1,2,3,4,5,6,7,8,9\}, A_{1}=\{1,2,4,5,7,8\}, A_{2}=\{2,3,5,6,8,9\}, B_{1}=$ $\{1,2,3,4,5,6\}, B_{2}=\{4,5,6,7,8,9\}, \mathcal{A}=\left\{A_{1}, A_{2}\right\}, \mathcal{B}=\left\{\varnothing, B_{1}, B_{2}\right\}$. Note that $\mathcal{S}_{\mathcal{A}}=\left\{A_{1}, A_{2}, X\right\}$ and $\mathcal{S}_{\mathcal{B}}=\left\{\varnothing, B_{1}, B_{2}, X\right\}$. First we will show that the family $\mathcal{S}_{\mathcal{A}} * \mathcal{S}_{\mathcal{B}}$ is not a semigroup of sets. It is enough to prove that the set $C=\left(\left(A_{1} \backslash B_{1}\right) \cup \varnothing\right) \cup\left(\left(A_{2} \backslash B_{2}\right) \cup \varnothing\right) \notin \mathcal{S}_{\mathcal{A}} * \mathcal{S}_{\mathcal{B}}$. It is clear that the set $C$ is given by $C=\left(A_{1} \backslash B_{1}\right) \cup\left(A_{2} \backslash B_{2}\right)=\{2,3,7,8\}$. Assume that $C \in \mathcal{S}_{\mathcal{A}} * \mathcal{S}_{\mathcal{B}}$. 
Thus $C=\left(\left(S_{1} \backslash S_{2}\right) \cup S_{3}\right)$ for some $S_{1} \in \mathcal{S}_{\mathcal{A}}$ and $S_{2}, S_{3} \in \mathcal{S}_{\mathcal{B}}$. Since $|C|=4$, we have $S_{3}=\varnothing$. Let $S_{1}=A_{1}$. Then $\left|S_{1} \backslash S_{2}\right|$ is either 2 (if $S_{2}$ is $B_{1}$ or $B_{2}$ ), 0 (if $S_{2}=X$ ) or 6 (if $S_{2}=\varnothing$ ). We have a contradiction. If $S_{1}=A_{2}$, we also have a contradiction by a similar argument as above. Assume now that $S_{1}=X$. Then $\left|S_{1} \backslash S_{2}\right|$ is either 3 (if $S_{2}$ is $B_{1}$ or $B_{2}$ ), 0 (if $S_{2}=X$ ) or 9 (if $S_{2}=\varnothing$ ). We have again a contradiction that proves the statement.

Further note that $\mathcal{S}_{\mathcal{B}} * \mathcal{S}_{\mathcal{A}}=\left\{A_{1}, A_{2},\{1\}^{c},\{3\}^{c},\{7\}^{c},\{9\}^{c}, X\right\}=\mathcal{S}_{\mathcal{A}} \cup \mathcal{S}_{\mathcal{B}}$. Hence, the family $\mathcal{S}_{\mathcal{B}} * \mathcal{S}_{\mathcal{A}}$ is a semigroup of sets.

Remark 3.2. The binary operation $*$ is not commutative, neither is associative, in general. This can be observed from the following example.

Example 3.4

Let $A$ and $B$ be two non-empty subsets of $X$ such that $A \subsetneq B$. Consider the families of sets $\mathcal{A}=\{\varnothing, A\}, \mathcal{B}=\{B\}$ and $\mathcal{C}=\{\varnothing, X\}$. Then we have $\mathcal{A} * \mathcal{B}=$ $\{B\}$ while $\mathcal{B} * \mathcal{A}=\{B, B \backslash A\}$. It is clear also that $(\mathcal{A} * \mathcal{B}) * \mathcal{C}=\{\varnothing, B, X\}$ while $\mathcal{A} *(\mathcal{B} * \mathcal{C})=\{\varnothing, A, B, X\}$.

\subsection{Extension of a semigroup of sets via an ideal of sets}

In this section, we present a way of obtaining a new semigroup of sets from an old one by the use of an ideal of sets.

Proposition 3.2. Let $\mathcal{S}$ be a semigroup of sets and $\mathcal{I}$ be an ideal of sets. Then the family $\mathcal{S} * \mathcal{I}$ is a semigroup of sets.

Proof. Take two elements $U_{1}$ and $U_{2}$ of $\mathcal{S} * \mathcal{I}$. Then $U_{1}=\left(S_{1} \backslash I_{1}^{\prime}\right) \cup I_{1}^{\prime \prime}$ and $\left.U_{2}=\left(S_{2} \backslash I_{2}^{\prime}\right) \cup I_{2}^{\prime \prime}\right)$ for some sets $S_{i} \in \mathcal{S}$ and $I_{i}^{\prime}, I_{i}^{\prime \prime} \in \mathcal{I}$ where $i=1,2$. We need to show that the set $U=U_{1} \cup U_{2} \in \mathcal{S} * \mathcal{I}$. In fact $U=\left(\left(S_{1} \backslash I_{1}^{\prime}\right) \cup I_{1}^{\prime \prime}\right) \cup$ $\left(\left(S_{2} \backslash I_{2}^{\prime}\right) \cup I_{2}^{\prime \prime}\right)=\left(S_{1} \backslash I_{1}^{\prime}\right) \cup\left(S_{2} \backslash I_{2}^{\prime}\right) \cup\left(I_{1}^{\prime \prime} \cup I_{2}^{\prime \prime}\right)$. Since $\mathcal{I}$ is an ideal of sets, then the set $I_{2}=I_{1}^{\prime \prime} \cup I_{2}^{\prime \prime} \in \mathcal{I}$. It follows that $U=\left(\left(S_{1} \cap I_{1}^{\prime c}\right) \cup\left(S_{2} \cap I_{2}^{\prime c}\right)\right)^{c c} \cup I_{2}=$ 
$\left(\left(S_{1} \cap I_{1}^{\prime c}\right)^{c} \cap\left(S_{2} \cap I_{2}^{\prime c}\right)^{c}\right)^{c} \cup I_{2}=\left(\left(S_{1}^{c} \cup I_{1}^{\prime}\right) \cap\left(S_{2}^{c} \cup I_{2}^{\prime}\right)\right)^{c} \cup I_{2}=\left(\left(S_{1}^{c} \cap S_{2}^{c}\right) \cup\left(S_{1}^{c} \cap\right.\right.$ $\left.\left.I_{2}^{\prime}\right) \cup\left(S_{2}^{c} \cap I_{1}^{\prime}\right) \cup\left(I_{1}^{\prime} \cap I_{2}^{\prime}\right)\right)^{c} \cup I_{2}$. Put $I_{1}=\left(S_{1}^{c} \cap I_{2}^{\prime}\right) \cup\left(S_{2}^{c} \cap I_{1}^{\prime}\right) \cup\left(I_{1}^{\prime} \cap I_{2}^{\prime}\right)$ and note that $U=\left(\left(S_{1}^{c} \cap S_{2}^{c}\right)^{c} \cap I_{1}^{c}\right) \cup I_{2}=\left(\left(S_{1} \cup S_{2}\right) \cap I_{1}^{c}\right) \cup I_{2}=\left(\left(S_{1} \cup S_{2}\right) \backslash I_{1}\right) \cup I_{2}$. It is easy to see that $S_{1} \cup S_{2} \in \mathcal{S}$ and $I_{1}, I_{2} \in \mathcal{I}$. Hence, $U \in \mathcal{S} * \mathcal{I}$.

The following proposition shows the relationship in the sense of inclusion between the defined operations when they are applied on semigroups of sets and ideals of sets.

Proposition 3.3. Let $\mathcal{S}$ be a semigroup of sets and $\mathcal{I}$ be an ideal of sets. Then

(a) $\mathcal{S} * \mathcal{I}=\mathcal{S} \Delta \mathcal{I} \supset \mathcal{S} \cup \mathcal{I}=\mathcal{I} * \mathcal{S} \supset \mathcal{S}$

(b) $(\mathcal{S} * \mathcal{I}) * \mathcal{I}=\mathcal{S} * \mathcal{I}, \mathcal{I} *(\mathcal{I} * \mathcal{S})=\mathcal{I} * \mathcal{S}$.

Proof. (a) Note that for any set $S \in \mathcal{S}$ and for any set $I \in \mathcal{I}$ we have $S \Delta I=$ $(S \backslash I) \cup(I \backslash S) \in \mathcal{S} * \mathcal{I}, S \cup I=S \Delta(I \backslash S) \in \mathcal{S} \Delta \mathcal{I}, S \cup I=(I \backslash S) \cup S \in \mathcal{I} * \mathcal{S}$ and $S=S \cup \varnothing \in \mathcal{S} \cup \mathcal{I}$. Thus, $\mathcal{S} * \mathcal{I} \supset \mathcal{S} \Delta \mathcal{I} \supset \mathcal{S} \cup \mathcal{I} \supset \mathcal{S}$ and $\mathcal{I} * \mathcal{S} \supset \mathcal{S} \cup \mathcal{I}$. Observe also that for any sets $S_{1}, S_{2} \in \mathcal{S}$ and any sets $I_{1}, I_{2} \in \mathcal{I}$ we have $\left(S_{1} \backslash I_{1}\right) \cup I_{2}=$ $S_{1} \Delta I \in \mathcal{S} \Delta \mathcal{I}$, where $I=\left(\left(I_{1} \cap S_{1}\right) \backslash I_{2}\right) \cup\left(I_{2} \backslash S_{1}\right)$, and $\left(I_{1} \backslash S_{1}\right) \cup S_{2} \in \mathcal{S} \cup \mathcal{I}$. There by, $\mathcal{S} * \mathcal{I} \subset \mathcal{S} \Delta \mathcal{I}$ and $\mathcal{I} * \mathcal{S} \subset \mathcal{S} \cup \mathcal{I}$.

(b) Let $S \in \mathcal{S}$ and $I_{1}, I_{2}, I_{3}, I_{4} \in \mathcal{I}$. Observe that $\left(\left(\left(S \backslash I_{1}\right) \cup I_{2}\right) \backslash I_{3}\right) \cup I_{4}=$ $\left(S \backslash\left(I_{1} \cup I_{3}\right)\right) \cup\left(\left(I_{2} \backslash I_{3}\right) \cup I_{4}\right) \in \mathcal{S} * \mathcal{I}$. Hence $(\mathcal{S} * \mathcal{I}) * \mathcal{I} \subset \mathcal{S} * \mathcal{I}$. The opposite inclusion is evident.

Let $I_{1}, I_{2}, I_{3} \in \mathcal{I}$ and $S_{1}, S_{2}, S_{3}, S_{4} \in \mathcal{S}$. Note that $\left(I_{1} \backslash\left(\left(I_{2} \backslash S_{1}\right) \cup S_{2}\right)\right) \cup$ $\left(\left(I_{3} \backslash S_{3}\right) \cup S_{4}\right)=\left(\left(I_{1} \backslash\left(\left(I_{2} \backslash S_{1}\right) \cup S_{2}\right)\right) \cup\left(I_{3} \backslash S_{3}\right)\right) \cup S_{4}=I \cup S_{4} \in \mathcal{I} * \mathcal{S}$, where $\left.I=\left(I_{1} \backslash\left(\left(I_{2} \backslash S_{1}\right)\right) \cup S_{2}\right)\right) \cup\left(I_{3} \backslash S_{3}\right)$. Hence $\mathcal{I} *(\mathcal{I} * \mathcal{S}) \subset \mathcal{I} * \mathcal{S}$. The opposite inclusion is evident.

Corollary 3.1. Let $\mathcal{S}$ be a semigroup of sets and $\mathcal{I}$ be an ideal of sets. Then

(a) The families $\mathcal{S} \Delta \mathcal{I}, \mathcal{I} * \mathcal{S}$ are semigroups of sets;

(b) $(\mathcal{I} * \mathcal{S}) * \mathcal{I}=\mathcal{I} *(\mathcal{S} * \mathcal{I})=\mathcal{S} * \mathcal{I}$

Proof. As $\mathcal{S} \Delta \mathcal{I}=\mathcal{S} * \mathcal{I}$, by Proposition 3.2, the family $\mathcal{S} \Delta \mathcal{I}$ is a semigroup of sets. From the observation (i) made above and the equality $\mathcal{S} \cup \mathcal{I}=\mathcal{I} * \mathcal{S}$, the family $\mathcal{I} * \mathcal{S}$ is also a semigroup of sets. This proves item (a). The item (b) follows by observing that $\mathcal{S} * \mathcal{I}=(\mathcal{S} * \mathcal{I}) * \mathcal{I} \supset(\mathcal{I} * \mathcal{S}) * \mathcal{I} \supset \mathcal{S} * \mathcal{I}$ and $\mathcal{S} * \mathcal{I}=(\mathcal{S} * \mathcal{I}) * \mathcal{I} \supset \mathcal{I} *(\mathcal{S} * \mathcal{I}) \supset \mathcal{S} * \mathcal{I}$. 
The following statement is evident.

Corollary 3.2. Let $\mathcal{I}_{1}, \mathcal{I}_{2}$ be ideals of sets. Then the family $\mathcal{I}_{1} * \mathcal{I}_{2}$ is an ideal of sets. Moreover, $\mathcal{I}_{1} * \mathcal{I}_{2}=\mathcal{I}_{2} * \mathcal{I}_{1}=\mathcal{I}_{1} \Delta \mathcal{I}_{2}=\mathcal{I}_{1} \cup \mathcal{I}_{2}$.

\section{Example 3.5}

Let $X=\{1,2\}, A=X, B=\{1\}, C=\{2\}, \mathcal{A}=\{A\}, \mathcal{B}=\{B\}$. Note that $\mathcal{S}_{\mathcal{A}}=\{A\}, \mathcal{S}_{\mathcal{B}}=\{B\}, \mathcal{I}_{\mathcal{B}}=\{\varnothing, B\}, \mathcal{S}_{\mathcal{A}} * \mathcal{I}_{\mathcal{B}}=\{A, C\}$ and $\mathcal{I}_{\mathcal{B}} * \mathcal{S}_{\mathcal{A}}=\{A\}$. Thus, in general, none of the following statements is valid: $\mathcal{S} * \mathcal{I}=\mathcal{I} * \mathcal{S}, \mathcal{S} * \mathcal{I} \supset \mathcal{I}$, the family $\mathcal{S} * \mathcal{I}$ is an ideal of sets or $\mathcal{I} * \mathcal{S}$ is an ideal of sets, where $\mathcal{S}$ is a semigroup of sets and $\mathcal{I}$ is an ideal of sets.

For two subfamilies $\mathcal{A}$ and $\mathcal{B}$ of $\mathcal{P}(X)$, put $\mathcal{A} \cap \mathcal{B}=\{Y: Y \in \mathcal{A}$ and $Y \in \mathcal{B}\}$.

The next statement is useful in the search of pairs of semigroups of sets without common elements.

Proposition 3.4. Let $\mathcal{I}$ be an ideal of sets and $\mathcal{A}, \mathcal{B} \subset \mathcal{P}(X)$ such that:

(a) $\mathcal{A} \cap \mathcal{I}=\varnothing$ (i.e. $\mathcal{A}$ and $\mathcal{I}$ have no common element);

(b) For each element $U \in \mathcal{S}_{\mathcal{A}}$ and each non-empty element $B \in \mathcal{B}$ there is an element $A \in \mathcal{A}$ such that $A \subset B \backslash U$.

Then

(1) For each element $I \in \mathcal{I}$, each element $U \in \mathcal{S}_{\mathcal{A}}$ and each non empty element $B \in \mathcal{B}$ we have $(U \cup I)^{c} \cap B \neq \varnothing$;

(2) For each elements $I_{1}, I_{2} \in \mathcal{I}$, each element $U \in \mathcal{S}_{\mathcal{A}}$ and each non-empty $B \in \mathcal{B}$ we have $\left(U \cup I_{1}\right)^{\mathcal{C}} \cap\left(B \backslash I_{2}\right) \neq \varnothing$;

(3) For each elements $I_{1}, I_{2}, I_{3}, I_{4} \in \mathcal{I}$, each element $U \in \mathcal{S}_{\mathcal{A}}$ and each element $V \in \mathcal{S}_{\mathcal{B}}$ we have $\left(U \backslash I_{1}\right) \cup I_{2} \neq\left(V \backslash I_{3}\right) \cup I_{4}$, i.e. $\left(\mathcal{S}_{\mathcal{A}} * \mathcal{I}\right) \cap\left(\mathcal{S}_{\mathcal{B}} * \mathcal{I}\right)=\varnothing$.

Proof. (1) Assume that $U \cup I \supset B$ for some non-empty element $B \in \mathcal{B}$. By (b) there is $A \in \mathcal{A}$ such that $A \subset B \backslash U$. Note that $A \subset(U \cup I) \backslash U \subset I$. But this is a contradiction with (a).

(2) Assume that $U \cup I_{1} \supset B \backslash I_{2}$ for some non-empty element $B \in \mathcal{B}$ and some element $I_{2} \in \mathcal{I}$. Note that $U \cup\left(I_{1} \cup I_{2}\right)=\left(U \cup I_{1}\right) \cup I_{2} \supset\left(B \backslash I_{2}\right) \cup I_{2} \supset B$. But this is a contradiction with (1). 
(3) Assume that $\left(U \backslash I_{1}\right) \cup I_{2}=\left(V \backslash I_{3}\right) \cup I_{4}$ for some elements $U \in \mathcal{S}_{\mathcal{A}}, V \in \mathcal{S}_{\mathcal{B}}$ and $I_{3}, I_{4} \in \mathcal{I}$. If $V=\varnothing$, then $\left(U \backslash I_{1}\right) \cup I_{2}=I_{4}$ and so $U \subset I_{1} \cup I_{4}$. But this is a contradiction with with (a). Hence $V \neq \varnothing$. Note that there is a non-empty element $B \in \mathcal{B}$ such that $B \subset V$. Further observe that $U \cup I_{2} \supset$ $\left(U \backslash I_{1}\right) \cup I_{2}=\left(V \backslash I_{3}\right) \cup I_{4} \supset B \backslash I_{3}$. But this is a contradiction with (2). 



\section{4}

\section{Semigroups of sets defined by Vitali selectors on the real line}

In this chapter, we present diverse semigroups of sets on the real line whose elements do not possess the Baire property. These semigroups will be constructed by the use of a concept of Vitali selectors rigorously defined in the coming section. The Vitali selectors are closely related to the classical Vitali sets on the real line (one should to replace the rationals $Q$ by any countable dense subgroup $Q$ of the reals $\mathbb{R}$ in the Vitali construction). They were considered in [3] and were called $\Gamma$-selectors of $\mathbb{R}$ there. It is known (cf. [3]) that each Vitali set (even each Vitali selector) of $\mathbb{R}$ does not possess the Baire property. This result was extended by V.A. Chatyrko [5] who showed that finite unions of Vitali sets on the real line also do not possess the Baire property. Furthermore, he observed that the family $\mathcal{S}_{\mathcal{V}(\mathrm{Q})}$ of all finite unions of Vitali sets, as well as the family $\mathcal{S}_{\mathcal{V}(\mathbb{Q})} * \mathcal{M}$, where $\mathcal{M}$ is the family of all meager sets on the real line, are semigroups of sets, invariant under translations of $\mathbb{R}$, and the elements of $\mathcal{S}_{\mathcal{V}(\mathbb{Q})} * \mathcal{M}$ also do not possess the Baire property.

So in this chapter, we generalize Chatyrko's results via replacing the Vitali sets by Vitali $Q$-selectors, where $Q$ is a countable dense subgroup $Q$ of $\mathbb{R}$, and the family $\mathcal{M}$ by any ideal of sets on $\mathbb{R}$ in the formulas $\mathcal{S}_{\mathcal{V}(\mathbb{Q})}$ and $\mathcal{S}_{\mathcal{V}(\mathbb{Q})} * \mathcal{M}$. We study the relationship between the semigroups for different $Q$ in the sense of inclusion. We observe that in the family of all semigroups of sets $\mathcal{S}_{\mathcal{V}(Q)}$, where $Q$ is varied, there is no element which contains all others. Furthermore we 
consider the family $\mathcal{V}^{\text {sup }}$ of all Vitali selectors of $\mathbb{R}$ and the family $\mathcal{S}_{\mathcal{V}}$ sup which we call the supersemigroup of sets based on Vitali selectors. We will show that the supersemigroup $\mathcal{S}_{\mathcal{V} \text { sup }}$ also consists of sets without the Baire property and is invariant under translations of $\mathbb{R}$. Let us note that the semigroup $\mathcal{S}_{\mathcal{V} \text { sup }}$ contains all semigroups $\mathcal{S}_{\mathcal{V}(Q)}$.

The results of this chapter were mostly taken from the articles [4] and [6].

\subsection{Vitali selectors of the real line}

Let $\mathbb{R}$ be the real line and $Q$ be a countable, dense in the real line subgroup of $(\mathbb{R},+)$. For an element $x \in \mathbb{R}$, denote by $T_{x}$ the translation of $\mathbb{R}$ by $x$, i.e. $T_{x}(y)=y+x$ for each element $y \in \mathbb{R}$. If $A$ is a subset of $\mathbb{R}$ and $x \in \mathbb{R}$, we denote $T_{x}(A)$ by $A_{x}$. Define the equivalence relation $E$ on $\mathbb{R}$ as follows: for $x, y \in \mathbb{R}$, let $x E y$ if and only if $x-y \in Q$ and let $E_{\alpha}(Q), \alpha \in I$ be the equivalence classes. Observe that $|I|=\mathfrak{c}$, where $\mathfrak{c}$ is the cardinality of the continuum, and for each index $\alpha \in I$ and each $x \in E_{\alpha}(Q)$ we have $E_{\alpha}(Q)=Q_{x}$. So, each equivalence class $E_{\alpha}(Q)$ is dense in $\mathbb{R}$.

Definition 4.1. A Vitali $Q$-selector (shortly, a Vitali selector) of $\mathbb{R}$ is any subset $V$ of $\mathbb{R}$ such that $\left|V \cap E_{\alpha}(Q)\right|=1$ for each $\alpha \in I$.

Note that a Vitali $Q$-selector is a Vitali set [2], if $Q$ is the set $\mathbb{Q}$ of rational numbers.

We continue with simple facts about Vitali selectors.

Proposition 4.1. Let $V$ be a Vitali $Q$-selector of $\mathbb{R}$.

(i) If $q_{1}, q_{2} \in Q$ and $q_{1} \neq q_{2}$ then $V_{q_{1}} \cap V_{q_{2}}=\varnothing$.

(ii) $\mathbb{R}=\bigcup_{q \in Q} V_{q}$.

(iii) The set $V$ is not meager in $\mathbb{R}$.

Proof. (i) Let $x \in V_{q_{1}} \cap V_{q_{2}}$ with $q_{1}, q_{2} \in Q$ and $q_{1} \neq q_{2}$. Then $x$ can be represented in two ways: $x=y+q_{1}=z+q_{2}$ for some $y, z \in V$. But $y-z=q_{2}-q_{1} \in Q$ implies that $y$ and $z$ are in the same equivalence class. Since $\left|V \cap E_{\alpha}(Q)\right|=1$ for all $\alpha \in I$, then $y=z$. This implies that $q_{1}=q_{2}$. We have a contradiction. 
(ii) If $x \in \mathbb{R}$, then $x$ belongs to a unique equivalence class $E_{\alpha}(Q)$. Let $v_{\alpha}$ be the representatative of $E_{\alpha}(Q)$ in $V$, i.e. $V \cap E_{\alpha}(Q)=\left\{v_{\alpha}\right\}$. So, $x-v_{\alpha}=q$ for some $q \in Q$. It follows that $x=v_{\alpha}+q \in V_{q}$.

(iii) If $V$ is a meager in $\mathbb{R}$, then each $V_{q}, q \in Q$, is a meager subset of $\mathbb{R}$, as a translation is a homeomorphism. This implies that the real line $\mathbb{R}$ is covered by countably many meager sets, and hence it is meager. This is in a contradiction with the Baire Category Theorem.

Lemma 4.1. For each Vitali $Q$-selector $V$ of $\mathbb{R}$ and each element $x \in \mathbb{R}$, the set $V_{x}$ is also a Vitali $Q$-selector of $\mathbb{R}$.

Proof. Let $V$ be an arbitrary Vitali $Q$-selector and let $x \in \mathbb{R}$.

Since for any different elements $v_{1}, v_{2}$ of $V$, we have $\left(v_{1}+x\right)-\left(v_{2}+x\right)=$ $v_{1}-v_{2} \in \mathbb{R} \backslash Q$, the elements $v_{1}+x$ and $v_{2}+x$ belong to different equivalence classes $E_{\alpha}, \alpha \in I$.

Consider a fixed $\alpha \in I$ and let $v_{\alpha}$ be the element in $V$ satisfying $\left\{v_{\alpha}\right\}=$ $E_{\alpha}(Q) \cap V$. So $v_{\alpha}-x \in E_{\beta}(Q)$ for some $\beta \in I$. Note that there is one element $v_{\beta}$ in $V$ such that $\left\{v_{\beta}\right\}=V \cap E_{\beta}(Q)$. Since $v_{\alpha}-x$ and $v_{\beta}$ belong to the same equivalence class $E_{\beta}(Q)$, there is a $q \in Q$ such that $v_{\alpha}-x-v_{\beta}=q$. So $v_{\beta}+x=$ $v_{\alpha}-q \in E_{\alpha}(Q)$. As $v_{\beta}+x \in V_{x}$, we have $\left|V_{x} \cap E_{\alpha}(Q)\right|=1$ for all $\alpha \in I$.

The family of all Vitali $Q$-selectors of $\mathbb{R}$ associated to the subgroup $Q$ will be denoted by $\mathcal{V}(Q)$ and $\mathcal{S}_{\mathcal{V}(Q)}$ will denote the semigroup of sets generated by $\mathcal{V}(Q)$ (Chapter 3, Section 3.1).

Proposition 4.2. The families $\mathcal{V}(Q)$ and $\mathcal{S}_{\mathcal{V}(Q)}$ are invariant under translations of $\mathbb{R}$.

Proof. Let $V \in \mathcal{V}(Q)$. By Lemma 4.1, it follows that for each element $x \in \mathbb{R}$, we have $V_{x} \in \mathcal{V}(Q)$. So, the family $\mathcal{V}(Q)$ is invariant under translations of $\mathbb{R}$. Since the family $\mathcal{S}_{\mathcal{V}(Q)}$ consists of all finite unions of elements of $\mathcal{V}(Q)$, then it is also invariant under translations of $\mathbb{R}$.

Lemma 4.2. For each $U \in \mathcal{S}_{\mathcal{V}(Q)}$ and each non-empty open set $O$ of $\mathbb{R}$, there is an element $V \in \mathcal{V}(Q)$ such that $V \subset O \backslash U$.

Proof. Let $U \in \mathcal{S}_{\mathcal{V}(Q)}$ and $O$ be a non-empty open set of $\mathbb{R}$. So $U=\bigcup_{i=1}^{n} V_{i}$ where $V_{i} \in \mathcal{V}(Q)$. To continue with the proof, first we show the following useful claim. 
Claim 4.1.1. For each element $\alpha \in I$, we have $E_{\alpha}(Q) \cap(O \backslash U) \neq \varnothing$.

Proof. From the density of each equivalence class $E_{\alpha}(Q)$ in the real line, we have $\left|E_{\alpha}(Q) \cap O\right|=\aleph_{0}$ for all $\alpha \in I$. By the definition of a Vitali $Q$-selector, we have $\left|E_{\alpha}(Q) \cap U\right|=\left|E_{\alpha}(Q) \cap\left(\bigcup_{i=1}^{n} V_{i}\right)\right|=\left|\bigcup_{i=1}^{n}\left(E_{\alpha}(Q) \cap V_{i}\right)\right| \leq n$ for all $\alpha \in I$. These two facts show that $E_{\alpha}(Q) \cap(O \backslash U) \neq \varnothing$.

For each equivalence class $E_{\alpha}(Q), \alpha \in I$, choose one element $y_{\alpha}$ in the set $E_{\alpha}(Q) \cap(O \backslash U)$. The set $V$ of such elements $y_{\alpha}$ is a Vitali $Q$-selector of $\mathbb{R}$. Moreover, $V \subset O$ and $V \cap U=\varnothing$. Hence $V \subset O \backslash U$.

Let $\mathcal{O}$ be the family of all open subsets of $\mathbb{R}$. Note that $\mathcal{O}$ is a semigroup of sets and $\mathcal{S}_{\mathcal{O}}=\mathcal{O}$.

Proposition 4.3. Let $\mathcal{I}$ be an ideal of subsets of $\mathbb{R}$. Then the following statements hold.

(i) The families $\mathcal{S}_{\mathcal{V}(Q)}, \mathcal{I} * \mathcal{S}_{\mathcal{V}(Q)}$ and $\mathcal{S}_{\mathcal{V}(Q)} * \mathcal{I}$ are semigroups of sets such that $\mathcal{S}_{\mathcal{V}(Q)} \subset \mathcal{I} * \mathcal{S}_{\mathcal{V}(Q)} \subset \mathcal{S}_{\mathcal{V}(Q)} * \mathcal{I}$

(ii) If $\mathcal{V}(Q) \cap \mathcal{I}=\varnothing$, then $\left(\mathcal{S}_{\mathcal{V}(Q)} * \mathcal{I}\right) \cap(\mathcal{O} * \mathcal{I})=\varnothing$.

In particular, $\mathcal{S}_{\mathcal{V}(Q)} \cap(\mathcal{O} * \mathcal{I})=\varnothing$.

(iii) If $\mathcal{I}$ is invariant under translations of $\mathbb{R}$, then the families $\mathcal{I} * \mathcal{S}_{\mathcal{V}(Q)}$ and $\mathcal{S}_{\mathcal{V}(Q)} * \mathcal{I}$ are also invariant under translations of $\mathbb{R}$.

Proof. (i) The family $\mathcal{S}_{\mathcal{V}(Q)}$ is a semigroup of sets by Proposition 3.1. By Proposition 3.2 and Corollary 3.1, the families $\mathcal{S}_{\mathcal{V}(Q)} * \mathcal{I}$ and $\mathcal{I} * \mathcal{S}_{\mathcal{V}(Q)}$ are also semigroups of sets. The inclusion follows from Proposition 3.3.

(ii) To prove the equality $\left(\mathcal{S}_{\mathcal{V}(Q)} * \mathcal{I}\right) \cap(\mathcal{O} * \mathcal{I})=\varnothing$, we apply Proposition 3.4, together with Lemma 4.2. Namely, the families $\mathcal{A}$ and $\mathcal{B}$ in Proposition 3.4 are considered as the families $\mathcal{V}(Q)$ and $\mathcal{O}$, respectively, and Lemma 4.2 plays the same role as condition (b) in Proposition 3.4.

The particular case follows from the inclusion $\mathcal{S}_{\mathcal{V}(Q)} \subset \mathcal{S}_{\mathcal{V}(Q)} * \mathcal{I}$.

(iii) The invariance of the families $\mathcal{I} * \mathcal{S}_{\mathcal{V}(Q)}$ and $\mathcal{S}_{\mathcal{V}(Q)} * \mathcal{I}$ under translations of $\mathbb{R}$ follows from Proposition 4.2 and the assumption made on $\mathcal{I}$. 
Let $\mathcal{M}$ be the $\sigma$-ideal of meager sets in $\mathbb{R}$ and let $\mathcal{B}_{p}$ be the family of all subsets of $\mathbb{R}$ having the Baire property.

Observe that $\mathcal{V}(Q) \cap \mathcal{M}=\varnothing$ (Proposition 4.1 (iii)), $\mathcal{B}_{p}=\mathcal{O} * \mathcal{M}$ (see Definition 2.10) and $\mathcal{M}$ is invariant under translations of $\mathbb{R}$.

Corollary 4.1. The families $\mathcal{S}_{\mathcal{V}(Q)}, \mathcal{M} * \mathcal{S}_{\mathcal{V}(Q)}$ and $\mathcal{S}_{\mathcal{V}(Q)} * \mathcal{M}$ are semigroups of sets such that $\mathcal{S}_{\mathcal{V}(Q)} \subset \mathcal{M} * \mathcal{S}_{\mathcal{V}(Q)} \subset \mathcal{S}_{\mathcal{V}(Q)} * \mathcal{M}$. They are invariant under translations of $\mathbb{R}$, and consist of sets without the Baire property. In particular, the family $\mathcal{S}_{\mathcal{V}(Q)}$ consists of sets without the Baire property.

Proof. Note only that by Proposition 4.3 (ii), we have the equality $\left(\mathcal{S}_{\mathcal{V}(Q)} * \mathcal{M}\right) \cap(\mathcal{O} * \mathcal{M})=\varnothing$. Since $\mathcal{B}_{p}=\mathcal{O} * \mathcal{M}$, then $\mathcal{S}_{\mathcal{V}(Q)} * \mathcal{M} \subset \mathcal{P}(\mathbb{R}) \backslash \mathcal{B}_{p}$.

In particular, from the inclusion $\mathcal{S}_{\mathcal{V}(Q)} \subset \mathcal{S}_{\mathcal{V}(Q)} * \mathcal{M}$, it follows that $\mathcal{S}_{\mathcal{V}(Q)} \subset \mathcal{P}(\mathbb{R}) \backslash \mathcal{B}_{p}$

Let $\mathcal{I}_{f}$ (resp. $\mathcal{I}_{c}, \mathcal{I}_{c d}$ or $\mathcal{I}_{n}$ ) be the ideal of finite (resp. countable, closed and discrete or nowhere dense) subsets of $\mathbb{R}$. Recall that for these ideals of sets, we have the inclusions $\mathcal{I}_{f} \subsetneq \mathcal{I}_{c d} \subsetneq \mathcal{I}_{c} \subsetneq \mathcal{M}$ and $\mathcal{I}_{c d} \subsetneq \mathcal{I}_{n} \subsetneq \mathcal{M}$. Note that these ideals are invariant under translations of $\mathbb{R}$.

Corollary 4.2. Let $\mathcal{I}$ be $\mathcal{I}_{f}, \mathcal{I}_{c}, \mathcal{I}_{c d}$ or $\mathcal{I}_{n}$. Then the families $\mathcal{I} * \mathcal{S}_{\mathcal{V}(Q)}$ and $\mathcal{S}_{\mathcal{V}(Q)} * \mathcal{I}$ are semigroups of sets such that $\mathcal{S}_{\mathcal{V}(Q)} \subset \mathcal{I} * \mathcal{S}_{\mathcal{V}(Q)} \subset \mathcal{S}_{\mathcal{V}(Q)} * \mathcal{I}$. They are invariant under translations of $\mathbb{R}$ and consist of sets without the Baire property.

Proof. The results follows from Proposition 4.3 and the mentioned above inclusions.

Remark 4.1. From the inclusions $\mathcal{I}_{f} \subsetneq \mathcal{I}_{c d} \subsetneq \mathcal{I}_{c} \subsetneq \mathcal{M}$ and $\mathcal{I}_{c d} \subsetneq \mathcal{I}_{n} \subsetneq \mathcal{M}$, it follows also that:

(a) $\mathcal{S}_{\mathcal{V}(Q)} * \mathcal{I}_{f} \subset \mathcal{S}_{\mathcal{V}(Q)} * \mathcal{I}_{c d} \subset \mathcal{S}_{\mathcal{V}(Q)} * \mathcal{I}_{c} \subset \mathcal{S}_{\mathcal{V}(Q)} * \mathcal{M}$ and $\mathcal{S}_{\mathcal{V}(Q)} * \mathcal{I}_{c d} \subset \mathcal{S}_{\mathcal{V}(Q)} * \mathcal{I}_{n} \subset \mathcal{S}_{\mathcal{V}(Q)} * \mathcal{M}$

(b) $\mathcal{I}_{f} * \mathcal{S}_{\mathcal{V}(Q)} \subset \mathcal{I}_{c d} * \mathcal{S}_{\mathcal{V}(Q)} \subset \mathcal{I}_{\mathcal{c}} * \mathcal{S}_{\mathcal{V}(Q)} \subset \mathcal{M} * \mathcal{S}_{\mathcal{V}(Q)}$ and $\mathcal{I}_{c d} * \mathcal{S}_{\mathcal{V}(Q)} \subset \mathcal{I}_{n} * \mathcal{S}_{\mathcal{V}(Q)} \subset \mathcal{M} * \mathcal{S}_{\mathcal{V}(Q)}$

It is interesting to know which of the semigroups are equal. 


\section{Example 4.1}

Let $Q=\mathbb{Q}$. Observe that for each element $A \in \mathcal{S}_{\mathcal{V}(Q)} * \mathcal{I}_{f}$, we have $|A \cap Q|<\infty$.

In fact, if $A \in \mathcal{S}_{\mathcal{V}(Q)} * \mathcal{I}_{f}$ then $A=\left(U \backslash I_{1}\right) \cup I_{2}$ where $U \in \mathcal{S}_{\mathcal{V}(Q)}$ and $I_{1}, I_{2} \in \mathcal{I}_{f}$. Recall that $U=\bigcup_{i=1}^{n} V_{i}$, where $V_{i} \in \mathcal{V}(Q)$. Thus, $|A \cap Q|=\mid((U \backslash$ $\left.\left.I_{1}\right) \cup I_{2}\right) \cap Q|\leq|\left(U \cup I_{2}\right) \cap Q|=|(U \cap Q) \cup\left(I_{2} \cap Q\right)|\leq| U \cap Q|+| I_{2} \cap Q \mid \leq$ $n+\left|I_{2}\right|<\infty$.

This implies that the semigroups of sets $\mathcal{S}_{\mathcal{V}(Q)} * \mathcal{I}_{f}$ and $\mathcal{S}_{\mathcal{V}(Q)} * \mathcal{I}_{c d}$ are not equal. In fact, let $V \in \mathcal{V}(Q)$ and let $\mathbb{Z}$ be the set of all integers. Since $\mathbb{Z}$ is a closed and discrete subset of $\mathbb{R}$, we have $V \cup \mathbb{Z} \in \mathcal{S}_{\mathcal{V}(Q)} * \mathcal{I}_{c d}$. But $|(V \cup \mathbb{Z}) \cap Q|=\aleph_{0}$. So, by the above observation, $V \cup \mathbb{Z} \notin \mathcal{S}_{\mathcal{V}(Q)} * \mathcal{I}_{f}$.

Proposition 4.4. Let $\mathcal{I}$ be an ideal of sets such that $\mathcal{V}(Q) \cap \mathcal{I}=\varnothing$. Then each element of the family $\mathcal{S}_{\mathcal{V}(Q)} * \mathcal{I}$ is zero-dimensional. In particular, each element of the family $\mathcal{S}_{\mathcal{V}(\mathrm{Q})}$ is zero-dimensional.

Proof. Let $A \in \mathcal{S}_{\mathcal{V}(Q)} * \mathcal{I}$. Then $A=(U \backslash F) \cup E$ for some $U \in \mathcal{S}_{\mathcal{V}(Q)}$ and $E, F \in \mathcal{I}$. The equality $\mathcal{V}(Q) \cap \mathcal{I}=\varnothing$ implies that $A \neq \varnothing$. Since $\varnothing \neq A \subset \mathbb{R}$ then $0 \leq \operatorname{dim} A \leq 1$, by the monotonicity property of dimension.

Assume that $\operatorname{dim} A=1$. By the Brouwer-Dimension Theorem, there must exist a non-empty open set $O$ in $\mathbb{R}$ such that $O \subseteq A$. But $A=(U \backslash F) \cup E \subseteq$ $U \cup E$. By Lemma 4.2, there exists $V \in \mathcal{V}(Q)$ such that $V \subset O \backslash U$. So, $V \subset$ $O \backslash U \subseteq(U \cup E) \backslash U \subseteq E$ which implies that $V \in \mathcal{I}$. This is a contradiction. So $\operatorname{dim} A=0$.

The particular case follows from the inclusion $\mathcal{S}_{\mathcal{V}(Q)} \subset \mathcal{S}_{\mathcal{V}(Q)} * \mathcal{I}$.

Corollary 4.3. Each element of the family $\mathcal{S}_{\mathcal{V}(Q)} * \mathcal{M}$ is zero-dimensional.

Proof. The statement follows from Proposition 4.4.

\subsection{Countable dense subgroups of $\mathbb{R}$ and generated semigroups}

Let $Q_{1}$ and $Q_{2}$ be subgroups of $(\mathbb{R},+)$. Define $Q_{1}+Q_{2}=\left\{q_{1}+q_{2}: q_{i} \in Q_{i}, i=\right.$ $1,2\}$. We observe that the sum $Q_{1}+Q_{2}$ is a subgroup of $(\mathbb{R},+)$ and both $Q_{1}$ and $Q_{2}$ are subgroups of $Q_{1}+Q_{2}$. Moreover, 
(i) If each $Q_{i}, i=1,2$ is countable then the subgroup $Q_{1}+Q_{2}$ is countable;

(ii) If one of the subgroups $Q_{i}, i=1,2$ is dense then so is $Q_{1}+Q_{2}$.

Let $\mathcal{F}$ be the family of all countable, dense in the real line subgroups of the additive group $(\mathbb{R},+)$.

Proposition 4.5. For each $Q_{1} \in \mathcal{F}$, there is a $Q_{2} \in \mathcal{F}$ such that $Q_{1} \subsetneq Q_{2}$.

Proof. Let $Q_{1} \in \mathcal{F}$. Since $Q_{1}$ is a countable subset of $\mathbb{R}$ and the set $\mathbb{R}$ is uncountable, we have $\mathbb{R} \backslash Q_{1} \neq \varnothing$. Consider an element $x \in \mathbb{R} \backslash Q_{1}$ and set $Q_{2}=Q_{1}+x \mathbb{Z}=\left\{q+n x: q \in Q_{1}, n \in \mathbb{Z}\right\}$, where $\mathbb{Z}$ is the additive group of all integers. It is clear that $Q_{2}$ is a countable subgroup of $(\mathbb{R},+)$ and $Q_{1} \subset Q_{2}$. The subgroup $Q_{2}$ is dense on $\mathbb{R}$ (it contains a dense subset $Q_{1}$ of $\mathbb{R}$ ) and hence $Q_{2} \in \mathcal{F}$. Moreover, $Q_{1} \subsetneq Q_{2}$, since $x \in Q_{2} \backslash Q_{1}$.

Proposition 4.6. Let $Q_{1}, Q_{2}$ be elements of $\mathcal{F}$ such that $Q_{1} \subsetneq Q_{2}$.

Then $\mathcal{S}_{\mathcal{V}\left(Q_{1}\right)} \cap \mathcal{V}\left(Q_{2}\right)=\varnothing$. In particular, $\mathcal{V}\left(Q_{1}\right) \cap \mathcal{V}\left(Q_{2}\right)=\varnothing$.

Proof. Assume that there exists $V \in \mathcal{S}_{\mathcal{V}\left(Q_{1}\right)} \cap \mathcal{V}\left(Q_{2}\right)$. Let $E_{\alpha}\left(Q_{2}\right)$ be an equivalence class with the respect to the subgroup $Q_{2}$. So,

$$
\left|V \cap E_{\alpha}\left(Q_{2}\right)\right|=1
$$

Since $Q_{1} \subsetneq Q_{2}$, we have $\left|Q_{2} / Q_{1}\right|>1$, where $Q_{2} / Q_{1}$ is the factor group of $Q_{2}$ by $Q_{1}$. Note that $E_{\alpha}\left(Q_{2}\right)=\bigcup_{\beta \in A_{\alpha}} E_{\beta}\left(Q_{1}\right)$, where $E_{\beta}\left(Q_{1}\right)$ are distinct equivalence classes with the respect to the subgroup $Q_{1}$ and $\left|A_{\alpha}\right|=\left|Q_{2} / Q_{1}\right|>1$. It follows that $\left|V \cap E_{\alpha}\left(Q_{2}\right)\right|=\left|V \cap\left(\bigcup_{\beta \in A_{\alpha}} E_{\beta}\left(Q_{1}\right)\right)\right|=\left|\bigcup_{\beta \in A_{\alpha}}\left(V \cap E_{\beta}\left(Q_{1}\right)\right)\right|=$ $\sum_{\beta \in A_{\alpha}}\left|V \cap E_{\beta}\right|=\left|A_{\alpha}\right|>1$. This is in contradiction with the Equality 4.1. So, we must have $\mathcal{S}_{\mathcal{V}\left(Q_{1}\right)} \cap \mathcal{V}\left(Q_{2}\right)=\varnothing$.

The particular case follows the inclusion $\mathcal{V}\left(Q_{1}\right) \subset \mathcal{S}_{\mathcal{V}\left(Q_{1}\right)}$.

Remark 4.2. Let $Q_{1}, Q_{2}$ be elements of $\mathcal{F}$ such that $Q_{1} \subsetneq Q_{2}$ and $Q_{2} / Q_{1}$ be the factor group of $Q_{2}$ by $Q_{1}$. We have either $1<\left|Q_{2} / Q_{1}\right|<\infty$ or $\left|Q_{2} / Q_{1}\right|=\aleph_{0}$.

Proposition 4.7. For each $Q_{1} \in \mathcal{F}$ there is a $Q_{2} \in \mathcal{F}$ such that $Q_{1} \subsetneq Q_{2}$ and $\left|Q_{2} / Q_{1}\right|=\aleph_{0}$

Proof. Let $Q_{1} \in \mathcal{F}$. We can apply repeatedly the Proposition 4.5 to obtain a sequence of elements in $\mathcal{F}$ in the following way: 
Set $Q^{1}=Q_{1}$. By Proposition 4.5 we can get $Q^{2} \in \mathcal{F}$ such $Q^{1} \subsetneq Q^{2}$, where $Q^{2}=Q^{1}+x_{1} \mathbb{Z}, x_{1} \in \mathbb{R} \backslash Q^{1}$. In the same way we can get $Q^{3} \in \mathcal{F}$ such $Q^{2} \subsetneq Q^{3}$ where $Q^{3}=Q^{2}+x_{2} \mathbb{Z}, x_{2} \in \mathbb{R} \backslash Q^{2}$, and so on.

By this procedure, we get a sequence $\left\{Q_{k}\right\}_{k=1}^{\infty}$ of elements in $\mathcal{F}$ with $Q^{1} \subsetneq Q^{2} \subsetneq Q^{3} \subsetneq \cdots \subsetneq Q^{k-1} \subsetneq Q^{k} \subseteq \cdots$, where $Q^{k}$ at the kth step is given by $Q^{k}=Q^{k-1}+x_{k-1} \mathbb{Z}$ and $x_{k-1} \in \mathbb{R} \backslash Q^{k-1}$. The inequality $Q^{k} \neq Q^{k+1}$ is clear since $x_{k} \in Q^{k+1} \backslash Q^{k}$.

Put $Q_{2}=\bigcup_{k=1}^{\infty} Q^{k}$. It is evident that $Q_{2}$ is a subgroup of $(\mathbb{R},+)$. Besides that, $Q_{2}$ is countable (it is a countable union of countable sets) and dense on $\mathbb{R}$ (it contains dense subsets $Q^{k}, k=1,2, \cdots$ of $\mathbb{R}$ ). Hence $Q_{2} \in \mathcal{F}$.

To prove that $\left|Q_{2} / Q_{1}\right|=\aleph_{0}$, we will observe that for each pair of distinct elements $x_{n}$ and $x_{m}$ in the sequence $\left\{x_{k}\right\}_{k=1}^{\infty}$, we have $\left(Q_{1}+x_{n}\right) \cap\left(Q_{1}+x_{m}\right)=\varnothing$.

For, let $y \in\left(Q_{1}+x_{n}\right) \cap\left(Q_{1}+x_{m}\right)$ for some $n>m$. Then $y=q_{1}+x_{n}=q_{2}+$ $x_{m}$ for some $q_{1}, q_{2} \in Q_{1}$. So $x_{n}=\left(q_{2}-q_{1}\right)+x_{m} \in Q_{1}+x_{m} \subset Q_{1}+x_{m} \mathbb{Z} \subset Q^{m+1}$. As $n>m$, we must have $x_{n} \in Q^{m+1} \subseteq Q^{n}$. But, by the construction $x_{n} \in \mathbb{R} \backslash Q^{n}$. We have a contradiction. So $\left(Q_{1}+x_{n}\right) \cap\left(Q_{1}+x_{m}\right)=\varnothing$.

This observation implies that $Q_{1}+x_{1}, Q_{1}+x_{2}, \ldots, Q_{1}+x_{k}, \ldots$ are different elements of $Q_{2} / Q_{1}$. Hence, $\left|Q_{2} / Q_{1}\right|=\aleph_{0}$.

Proposition 4.8. Let $Q_{1}, Q_{2}$ be elements of $\mathcal{F}$ such that $Q_{1} \subsetneq Q_{2}$ and $\left|Q_{2} / Q_{1}\right|=\aleph_{0}$. Then $\mathcal{S}_{\mathcal{V}\left(Q_{1}\right)} \cap \mathcal{S}_{\mathcal{V}\left(Q_{2}\right)}=\varnothing$.

Proof. Assume that there exists $U \in \mathcal{S}_{\mathcal{V}\left(Q_{1}\right)} \cap \mathcal{S}_{\mathcal{V}\left(Q_{2}\right)}$. Since $U \in \mathcal{S}_{\mathcal{V}\left(Q_{2}\right)}$, then $U$ can be represented as $U=\bigcup_{i=1}^{n} V_{i}$ where $V_{i} \in \mathcal{V}\left(Q_{2}\right)$ for all $i$.

Let $E_{\alpha}\left(Q_{2}\right)$ be an equivalence class with the respect to the subgroup $Q_{2}$. So,

$$
\left|U \cap E_{\alpha}\left(Q_{2}\right)\right| \leq n
$$

Since $\left|Q_{2} / Q_{1}\right|=\aleph_{0}$, we have $E_{\alpha}\left(Q_{2}\right)=\bigcup_{\beta \in A_{\alpha}} E_{\beta}\left(Q_{1}\right)$, where $E_{\beta}\left(Q_{1}\right)$ are distinct equivalence classes with the respect to the subgroup $Q_{1}$ and $\left|A_{\alpha}\right|=\aleph_{0}$.

Since $U \in \mathcal{S}_{\mathcal{V}\left(Q_{1}\right)}$ then $U$ can be also represented as $U=\bigcup_{k=1}^{m} W_{k}$ where $W_{k} \in \mathcal{V}\left(Q_{1}\right)$ for all $k$.

Let $W$ be an arbitrary Vitali $Q_{1}$-selector among $W_{i}$ 's making the union $U$. So $\left|U \cap E_{\alpha}\left(Q_{2}\right)\right| \geq\left|W \cap E_{\alpha}\left(Q_{2}\right)\right|=\left|W \cap\left(\bigcup_{\beta \in A_{\alpha}} E_{\beta}\left(Q_{1}\right)\right)\right|=\left|\bigcup_{\beta \in A_{\alpha}}\left(W \cap E_{\beta}\left(Q_{1}\right)\right)\right|=$ $\sum_{\beta \in A_{\alpha}}\left|W \cap E_{\beta}\left(Q_{1}\right)\right|=\left|A_{\alpha}\right|=\aleph_{0}$.

This is in contradiction with the Inequality 4.2. So $\mathcal{S}_{\mathcal{V}\left(Q_{1}\right)} \cap \mathcal{S}_{\mathcal{V}\left(Q_{2}\right)}=\varnothing$. 
Corollary 4.4. For each $Q_{1} \in \mathcal{F}$ there is a $Q_{2} \in \mathcal{F}$ such that $\mathcal{S}_{\mathcal{V}\left(Q_{2}\right)} \cap \mathcal{S}_{\mathcal{V}\left(Q_{1}\right)}=\varnothing$. In particular, the family $\left\{\mathcal{S}_{\mathcal{V}(Q)}: Q \in \mathcal{F}\right\}$ of all semigroups of Vitali selectors has no element which contains all others.

Proof. Let $Q_{1} \in \mathcal{F}$. By Proposition 4.7, one can find $Q_{2} \in \mathcal{F}$ such that $Q_{1} \subsetneq Q_{2}$ and $\left|Q_{2} / Q_{1}\right|=\aleph_{0}$. Then, Proposition 4.8 implies that $\mathcal{S}_{\mathcal{V}\left(Q_{2}\right)} \cap \mathcal{S}_{\mathcal{V}\left(Q_{1}\right)}=\varnothing$. So, there is no $Q^{*} \in \mathcal{F}$ such that the generated semigroup $\mathcal{S}_{\mathcal{V}\left(Q^{*}\right)}$ contains the semigroup $\mathcal{S}_{\mathcal{V}(Q)}$ for each $Q \in \mathcal{F}$.

\subsection{Supersemigroup based on Vitali selectors of $\mathbb{R}$}

In the previous section, we have observed that there is no $Q \in \mathcal{F}$ such that the corresponding generated semigroup $\mathcal{S}_{\mathcal{V}(Q)}$ contains all others.

Definition 4.2. Put $\mathcal{V}^{\text {sup }}=\{V: V \in \mathcal{V}(Q), Q \in \mathcal{F}\}$. The family $\mathcal{S}_{\mathcal{V} \text { sup }}$ is called the supersemigroup of sets based on Vitali selectors of $\mathbb{R}$.

For the family $\mathcal{S}_{\mathcal{V}}$ sup, we have the following observations:

(i) As each element of $\mathcal{V}^{\text {sup }}$ is invariant under translation of $\mathbb{R}$ (Proposition 4.2), the family $\mathcal{S}_{\mathcal{V}}$ sup is invariant under translations of $\mathbb{R}$.

(ii) For each $Q \in \mathcal{F}$ and each ideal of sets $\mathcal{I}$ on $\mathbb{R}$ the inclusions $\mathcal{V}(Q) \subset$ $\mathcal{V}^{\text {sup }}, \mathcal{S}_{\mathcal{V}(Q)} \subset \mathcal{S}_{\mathcal{V}^{\text {sup }},} \mathcal{I} * \mathcal{S}_{\mathcal{V}(Q)} \subset \mathcal{I} * \mathcal{S}_{\mathcal{V} \text { sup }}$ and $\mathcal{S}_{\mathcal{V}(Q)} * \mathcal{I} \subset \mathcal{S}_{\mathcal{V} \text { sup }} * \mathcal{I}$ evidently hold.

(iii) By Propositions 3.2 and Corollary 3.1, the families $\mathcal{S}_{\mathcal{V} \text { sup }} * \mathcal{I}$ and $\mathcal{I} * \mathcal{S}_{\mathcal{V} \text { sup }}$ are semigroups of sets, for each ideal of sets $\mathcal{I}$ on $\mathbb{R}$.

Lemma 4.3. For each set $U \in \mathcal{S}_{\mathcal{V}}$ sup and each non-empty open set $O$ of $\mathbb{R}$, there is a set $V \in \mathcal{V}^{\text {sup }}$ such that $V \subset O \backslash U$.

Proof. Let $U=\bigcup_{i=1}^{n} V_{i}$, where $V_{i} \in \mathcal{V}\left(Q_{i}\right)$ and $Q_{i} \in \mathcal{F}, i=1, \cdots, n$. Note that the statement is valid when $Q_{1}=\cdots=Q_{n}$ (Lemma 4.2). Now we will consider the general case. Put $Q=\sum_{i=1}^{n} Q_{i}=\left\{\sum_{i=1}^{n} q_{i}: q_{i} \in Q_{i}\right\}$ and note that $Q \in \mathcal{F}$.

Claim 4.3.1. For each $x \in \mathbb{R}$ we have $\left|Q_{x} \cap(O \backslash U)\right| \geq 1$.

In fact $\left|Q_{x} \cap(O \backslash U)\right|=\aleph_{0}$. 
Proof. For $n=1$ the statement evidently holds by Lemma 4.2. Let $n \geq 2$. Let $O_{i}, i \leq n$, be non-empty open sets of $\mathbb{R}$ such that $x+O_{1}+\cdots+O_{n}=$ $\left\{x+x_{1}+\cdots+x_{n}: x_{i} \in O_{i}, i \leq n\right\} \subset O$. For each $i \leq n$ choose $n+1$ different points $q_{i}(j), j \leq n+1$, of $O_{i} \cap Q_{i}$.

Let now $Q_{i}^{*}=\left\{q_{i}^{j}: j \geq 1\right\}, i=1 \leq n$, and $q_{i}^{j}=q_{i}(j), i \leq n ; j \leq n+1$. Observe that for each $i \leq n$ and each $j_{1}, \cdots \widehat{j_{i}}, \cdots, j_{n}$ (the notation $\widehat{a}$ means that $a$ is not there) the set $\left\{x+q_{1}^{j_{1}}+\cdots+q_{i}^{k}+\cdots+q_{n}^{j_{n}}: k \geq 1\right\}$ consists of countably many different points (a coset of $Q_{i}^{*}$ ) and only one of them belongs to $V_{i}$.

Consider now $n$-dimensional digital box $B=\left\{\left(j_{1}, \cdots, j_{n}\right): j_{i} \leq n+1, i \leq\right.$ $n\}$. Note that $|B|=(n+1)^{n}$ and call the elements of $B$ by cells. Put in each cell $\left(j_{1}, \cdots, j_{n}\right)$ of $B$ the sum $x+q_{1}^{j_{1}}+\cdots+q_{n}^{j_{n}}$.

Fix $i \leq n$ and observe that each interval $I\left(j_{1}, \cdots \widehat{j_{i}}, \cdots, j_{n}\right)=$ $\left\{\left(j_{1}, \cdots, k, \cdots, j_{n}\right): k \leq n+1\right\}$ of cells contains at most one element of $V_{i}$. So the whole box $B$ contains at most $(n+1)^{n-1}$ elements of $V_{i}$.

Summarizing we have at most $n(n+1)^{n-1}$ elements of $U$ in the box $B$. Since $(n+1)^{n}>n(n+1)^{n-1}$ for $n \geq 2$, there are points $p$ in $B$ which are not elements of $U$. But such $p$ must be element of the set $Q_{x} \cap O$ by our choice. The claim is proved.

Let us finish the proof of the Lemma. For each equivalence class $Q_{x}$ choose a point from the set $Q_{x} \cap(O \backslash U)$. The set of such points is a Vitali $Q$-selector $V$ of $\mathbb{R}$ such that $V \subset O \backslash U$.

The following Theorem summarizes our main results about the supersemigroup of sets based on Vitali selectors on the real line.

Theorem 4.1. Let $\mathcal{I}$ be an ideal of subsets of $\mathbb{R}$. Then the following statements hold.

(i) The families $\mathcal{S}_{\mathcal{V} \text { sup }}, \mathcal{I} * \mathcal{S}_{\mathcal{V}}$ sup and $\mathcal{S}_{\mathcal{V}}$ sup $* \mathcal{I}$ are semigroups of sets such that $\mathcal{S}_{\mathcal{V} \text { sup }} \subset \mathcal{I} * \mathcal{S}_{\mathcal{V} \text { sup }} \subset \mathcal{S}_{\mathcal{V}_{\text {sup }} * \mathcal{I}}$

(ii) If $\mathcal{V}^{\text {sup }} \cap \mathcal{I}=\varnothing$ then

(a) $\left(\mathcal{S}_{\mathcal{V} \text { sup }} * \mathcal{I}\right) \cap(\mathcal{O} * \mathcal{I})=\varnothing$.

In particular, $\mathcal{S}_{\mathcal{V} \text { sup }} \cap(\mathcal{O} * \mathcal{I})=\varnothing$.

(b) For each $A \in \mathcal{S}_{\mathcal{V}}$ sup $* \mathcal{I}$, we have $\operatorname{dim} A=0$.

In particular, for each element $A \in \mathcal{S}_{\mathcal{V}}$ sup, we have $\operatorname{dim} A=0$. 
(iii) If $\mathcal{I}$ is invariant under translations of $\mathbb{R}$, then the families $\mathcal{I} * \mathcal{S}_{\mathcal{V}}$ sup and $\mathcal{S}_{\mathcal{V}}$ sup $* \mathcal{I}$ are also invariant under translations of $\mathbb{R}$.

(iv) For each $Q \in \mathcal{F}$ we have $\mathcal{S}_{\mathcal{V}(Q)} * \mathcal{I} \subset \mathcal{S}_{\mathcal{V}}$ sup $* \mathcal{I}$.

Proof. (i) The families $\mathcal{S}_{\mathcal{V} \text { sup }}, \mathcal{S}_{\mathcal{V}}$ sup $* \mathcal{I}$ and $\mathcal{I} * \mathcal{S}_{\mathcal{V}}$ sup are semigroups of sets by Propositions 3.1, Proposition 3.2 and Corollary 3.1, respectively. The inclusions follow from Proposition 3.3.

(ii) (a) To prove the equality $\left(\mathcal{S}_{\mathcal{V} \text { sup }} * \mathcal{I}\right) \cap(\mathcal{O} * \mathcal{I})=\varnothing$, we apply Proposition 3.4 and Lemma 4.3: the families $\mathcal{A}$ and $\mathcal{B}$ in Proposition 3.4 are considered as the families $\mathcal{V}^{\text {sup }}$ and $\mathcal{O}$, respectively, and Lemma 4.3 plays the same role as condition (b) in Proposition 3.4. The particular case follows from the inclusion $\mathcal{S}_{\mathcal{V} \text { sup }} \subset \mathcal{S}_{\mathcal{V} \text { sup }} * \mathcal{I}$.

(b) Let $A \in \mathcal{S}_{\mathcal{V} \text { sup }} * \mathcal{I}$. Then $A=(U \backslash M) \cup N$ where $U \in \mathcal{S}_{\mathcal{V} \text { sup }}$ and $M, N \in \mathcal{I}$. The equality $\mathcal{V}^{\text {sup }} \cap \mathcal{I}=\varnothing$ implies that $A \neq \varnothing$. Since $A \subset \mathbb{R}$, we must have $0 \leq \operatorname{dim} A \leq 1$.

Assume that $\operatorname{dim} A=1$. By the Brouwer-Dimension Theorem, there must exist a non-empty open set $O$ in $\mathbb{R}$ such that $O \subseteq A$. But $A=(U \backslash M) \cup$ $N \subseteq U \cup N$. By Lemma 4.3, there exists $V \in \mathcal{V}^{\text {sup }}$ such that $V \subset O \backslash U$. So, $V \subset O \backslash U \subseteq(U \cup N) \backslash U \subseteq N$ which implies that $V$ is an element of $\mathcal{I}$. This is a contradiction. So $\operatorname{dim} A=0$. The particular case easily follows.

(iii) The invariance of the families under translations of $\mathbb{R}$ follows from Proposition 4.2 and the assumption that $\mathcal{I}$ is invariant under translations of $\mathbb{R}$.

(iv) The result was pointed out in the second observation at the beginning of this section.

Corollary 4.5. The families $\mathcal{S}_{\mathcal{V}}$ sup, $\mathcal{M} * \mathcal{S}_{\mathcal{V}}$ sup and $\mathcal{S}_{\mathcal{V}}$ sup $* \mathcal{M}$ are semigroups of sets such that $\mathcal{S}_{\mathcal{V}}$ sup $\subset \mathcal{M} * \mathcal{S}_{\mathcal{V} \text { sup }} \subset \mathcal{S}_{\mathcal{V}}$ sup $* \mathcal{M}$. They are invariant under translations of $\mathbb{R}$, and consist of sets without the Baire property. Moreover, for each $A \in \mathcal{S}_{\mathcal{V}}$ sup $* \mathcal{M}$, we have $\operatorname{dim} A=0$.

Proof. Let us observe that the equality $\mathcal{V}(Q) \cap \mathcal{M}=\varnothing$ for each $Q \in \mathcal{F}$ implies that $\mathcal{V}^{\text {sup }} \cap \mathcal{M}=\varnothing$. Keeping in mind that $\mathcal{B}_{p}=\mathcal{O} * \mathcal{M}$, from the equality 
$\left(\mathcal{S}_{\mathcal{V} \text { sup }} * \mathcal{M}\right) \cap(\mathcal{O} * \mathcal{M})=\varnothing$, it follows that $\mathcal{S}_{\mathcal{V} \text { sup }} * \mathcal{M} \subset \mathcal{P}(\mathbb{R}) \backslash \mathcal{B}_{p}$. The other statements of the corollary follow from Theorem 4.1.

Remark 4.3. In the previous Corollary we can substitute $\mathcal{M}$ by any ideal from the following list $\mathcal{I}_{f}, \mathcal{I}_{c}, \mathcal{I}_{c d}$ or $\mathcal{I}_{n}$.

\subsection{Semigroup of non-Lebesgue measurable sets}

In this section we consider Vitali sets on the real line. Using the operation $*$, we produce a semigroup consisting of sets which are not measurable in the Lebesgue sense.

In [15] Kharazishvili proved that each element $U$ of the family $\mathcal{S}_{\mathcal{V}(\mathbb{Q})}$ is nonmeasurable in the Lebesgue sense. Let $\mathcal{N}$ be the family of all measurable sets in the Lebesgue sense on the real line $\mathbb{R}$ and let $\mathcal{N}_{0}$ be the family of all sets of the Lebesgue measure zero. Recall that the family $\mathcal{N}_{0}$ is an ideal of sets (in fact, a $\sigma$-ideal). It follows from Proposition 3.2 and Proposition 3.3 that the families $\mathcal{S}_{\mathcal{V}(\mathrm{Q})}, \mathcal{N}_{0} * \mathcal{S}_{\mathcal{V}(\mathrm{Q})}$ and $\mathcal{S}_{\mathcal{V}(\mathrm{Q})} * \mathcal{N}_{0}$ are three different semigroups of sets invariant under translation of $\mathbb{R}$ and $\mathcal{S}_{\mathcal{V}(\mathrm{Q})} \subset \mathcal{N}_{0} * \mathcal{S}_{\mathcal{V}(\mathrm{Q})} \subset \mathcal{S}_{\mathcal{V}(\mathrm{Q})} * \mathcal{N}_{0}$.

We have the following generalization of Kharazishvili's result.

Proposition 4.9. Each element of the family $\mathcal{S}_{\mathcal{V}(\mathrm{Q})} * \mathcal{N}_{0}$ is nonmeasurable in the Lebesgue sense.

Proof. In fact, let $A \in \mathcal{S}_{\mathcal{V}(\mathrm{Q})} * \mathcal{N}_{0}$ and assume that $A \in \mathcal{N}$. By Proposition 3.3 there are an $U \in \mathcal{S}_{\mathcal{V}(\mathrm{Q})}$ and $N \in \mathcal{N}_{0}$ such that $A=U \Delta N$. It is known that if $A_{1}, A_{2}$ are sets such that $A_{1} \in \mathcal{N}$ and the set $A_{1} \Delta A_{2}$ is of the Lebesgue measure zero, then the set $A_{2}$ must belong to the family $\mathcal{N}$ [16]. But $A \Delta U=(U \Delta N) \Delta U=$ $N$, hence $U \in \mathcal{N}$. This is a contradiction of Kharazishvili's result. So we must have $A \notin \mathcal{N}$.

Question 4.4.1. Is each element $U$ of the family $\mathcal{S}_{\mathcal{V} \text { sup }} * \mathcal{N}_{0}$ nonmeasurable in the Lebesgue sense? 


\section{Semigroups of sets without the Baire}

\section{property in finite dimensional \\ Euclidean spaces}

In the previous chapter, we were concerned with semigroups of sets without the Baire property related to Vitali selectors of $\mathbb{R}$. In this chapter, we consider Vitali selectors of $\mathbb{R}^{n}, n \geq 2$. Unlike to the real line, for the Euclidean spaces $\mathbb{R}^{n}, n \geq 2$, besides ordinary Vitali selectors, there exist also special Vitali selectors called here rectangular Vitali selectors.

Analogously to Chapter 4, we present semigroups of sets without the Baire property generated by these two types of Vitali selectors of $\mathbb{R}^{n}, n \geq 2$, etc. Furthermore, we explore the role of dimension in defining semigroups of sets on $\mathbb{R}^{n}$ without the Baire property.

The results of this chapter were mostly taken from the articles [4] and [6].

\subsection{Vitali selectors of $\mathbb{R}^{n}$}

Let $n \geq 2$. Consider $\left(\mathbb{R}^{n},+\right)$ the additive group of real $n$-tupples and let $Q$ be a countable dense subgroup of $\left(\mathbb{R}^{n},+\right)$, where $\mathbb{R}^{n}$ is endowed with the Euclidean topology.

For $\bar{x} \in \mathbb{R}^{n}$, denote by $T_{\bar{x}}$ the translation of $\mathbb{R}^{n}$ by $\bar{x}$, i.e. $T_{\bar{x}}(\bar{y})=\bar{y}+\bar{x}$ for each $\bar{y} \in \mathbb{R}^{n}$. If $A$ is a subset of $\mathbb{R}^{n}$ and $\bar{x} \in \mathbb{R}^{n}$, we denote $T_{\bar{x}}(A)$ by $A_{\bar{x}}$. It is 
evident that if $A$ is the product $\prod_{j=1}^{n} A_{j}$ of $A_{j} \subset \mathbb{R}, j \leq n$ and $\bar{x}=\left(x_{1}, \cdots, x_{n}\right)$ then $A_{\bar{x}}=\prod_{j=1}^{n}\left(A_{j}\right)_{x_{j}}$.

If $\bar{x}, \bar{y} \in \mathbb{R}^{n}$, we say that " $\bar{x}$ is equivalent to $\bar{y}$ " and write $\bar{x} E \bar{y}$ if and only if $\bar{x}-\bar{y} \in Q$. It is clear that $E$ is an equivalence relation and hence divides $\mathbb{R}^{n}$ into a collection of disjoint equivalence classes $E_{\alpha}(Q), \alpha \in I$ with $|I|=\mathfrak{c}$, where $\mathfrak{c}$ is the cardinality of the continuum. Observe that for each $\alpha \in I$ and each $\bar{x} \in E_{\alpha}(Q)$ we have $E_{\alpha}(Q)=Q_{\bar{x}}$. As a translation is a homeomorphism, it follows that each equivalence class $E_{\alpha}(Q)$ is a dense subset of $\mathbb{R}^{n}$.

Definition 5.1. A Vitali $Q$-selector (shortly, a Vitali selector) of $\mathbb{R}^{n}$ is any subset $V$ of $\mathbb{R}^{n}$ satisfying the equality $\left|V \cap E_{\alpha}(Q)\right|=1$ for each $\alpha \in I$.

Like in the real line, for Vitali selectors in $\mathbb{R}^{n}, n \geq 2$, the following Proposition holds. It can be proved in the same way as Proposition 4.1.

Proposition 5.1. Let $V$ be a Vitali $Q$-selector of $\mathbb{R}^{n}, n \geq 2$.

(i) If $\bar{q}_{1}, \bar{q}_{2} \in Q$ and $\bar{q}_{1} \neq \bar{q}_{2}$ then $V_{\bar{q}_{1}} \cap V_{\bar{q}_{2}}=\varnothing$.

(ii) $\mathbb{R}^{n}=\bigcup_{\bar{q} \in Q} V_{\bar{q}}$.

(iii) The set $V$ is not meager in $\mathbb{R}^{n}$.

We continue with diverse facts about Vitali selectors of $\mathbb{R}^{n}, n \geq 2$.

Lemma 5.1. For each Vitali $Q$-selector of $\mathbb{R}^{n}$ and for each $\bar{x} \in \mathbb{R}^{n}$, the set $V_{\bar{x}}$ is also a Vitali $Q$-selector of $\mathbb{R}^{n}$.

Proof. To prove the Lemma, apply the same arguments like in Lemma 4.1 on the real line.

Denote by $\mathcal{V}^{n}(Q)$ the family of all Vitali $Q$-selectors of $\mathbb{R}^{n}, n \geq 2$ associated to the subgroup $Q$. Let $\mathcal{S}_{\mathcal{V}^{n}(Q)}$ be the semigroup of sets generated by the family $\mathcal{V}^{n}(Q)$ (Chapter 3, Section 3.1).

Proposition 5.2. The families $\mathcal{V}^{n}(Q)$ and $\mathcal{S}_{\mathcal{V}^{n}(Q)}$ are invariant under translations of $\mathbb{R}^{n}$.

Proof. Let $V \in \mathcal{V}^{n}(Q)$. Lemma 5.1 tells us that for each vector $\bar{x} \in \mathbb{R}^{n}$, the set $V_{\bar{x}}$ is also a Vitali $Q$-selector of $\mathbb{R}^{n}$, i.e. $V_{\bar{x}} \in \mathcal{V}^{n}(Q)$. Thus, the family $\mathcal{V}^{n}(Q)$ is invariant under translations of $\mathbb{R}^{n}$. The family $\mathcal{S}_{\mathcal{V}^{n}(Q)}$ is invariant under translations of $\mathbb{R}^{n}$ as it consists of all finite unions of elements in $\mathcal{V}^{n}(Q)$. 
Lemma 5.2. For any set $U \in \mathcal{S}_{\mathcal{V}^{n}(Q)}$ and any non-empty open set $O$ of $\mathbb{R}^{n}$, there is a set $V \in \mathcal{V}^{n}(Q)$ such that $V \subset O \backslash U$.

Proof. Let $U \in \mathcal{S}_{\mathcal{V}^{n}(Q)}$ and let $O$ be a non-empty open subset of $\mathbb{R}^{n}$. So $U=$ $\bigcup_{i=1}^{m} V_{i}$, where each $V_{i} \in \mathcal{V}^{n}(Q), m \in \mathbb{N}$. In a similar way as it was done on the real line in the Claim 4.1.1, we have $E_{\alpha}(Q) \cap(O \backslash U) \neq \varnothing$ for each $\alpha \in I$.

For each equivalence class $E_{\alpha}(Q), \alpha \in I$, choose one element $\bar{y}_{\alpha}$ in the set $E_{\alpha}(Q) \cap(O \backslash U)$. The set $V$ of such elements $\bar{y}_{\alpha}$ is a Vitali $Q$-selector of $\mathbb{R}^{n}$. Furthermore $V \subset O$ and $V \cap U=\varnothing$ by construction. Hence $V \subset O \backslash U$.

Let $\mathcal{O}^{n}$ be the family of all open subsets in the Euclidean topology of $\mathbb{R}^{n}$. Note that the family $\mathcal{O}^{n}$ is a semigroup of sets and $\mathcal{S}_{\mathcal{O}^{n}}=\mathcal{O}^{n}$.

Proposition 5.3. Let $\mathcal{I}$ be an ideal of subsets of $\mathbb{R}^{n}$. Then the following statements hold:

(i) The families $\mathcal{S}_{\mathcal{V}^{n}(Q)}, \mathcal{I} * \mathcal{S}_{\mathcal{V}^{n}(Q)}$ and $\mathcal{S}_{\mathcal{V}^{n}(Q)} * \mathcal{I}$ are semigroups of sets such that $\mathcal{S}_{\mathcal{V}^{n}(Q)} \subset \mathcal{I} * \mathcal{S}_{\mathcal{V}^{n}(Q)} \subset \mathcal{S}_{\mathcal{V}^{n}(Q)} * \mathcal{I}$

(ii) If $\mathcal{V}^{n}(Q) \cap \mathcal{I}=\varnothing$ then

(a) $\left(\mathcal{S}_{\mathcal{V}^{n}(Q)} * \mathcal{I}\right) \cap\left(\mathcal{O}^{n} * \mathcal{I}\right)=\varnothing$.

In particular, $\mathcal{S}_{\mathcal{V}^{n}(Q)} \cap\left(\mathcal{O}^{n} * \mathcal{I}\right)=\varnothing$.

(b) For each element $A$ of $\mathcal{S}_{\mathcal{V}^{n}(Q)} * \mathcal{I}$, we have $0 \leq \operatorname{dim} A \leq n-1$.

In particular, for each element $A$ of $\mathcal{S}_{\mathcal{V}^{n}(Q)}$, we have $0 \leq \operatorname{dim} A \leq n-1$.

(iii) If $\mathcal{I}$ is invariant under translations of $\mathbb{R}^{n}$, then $\mathcal{I} * \mathcal{S}_{\mathcal{V}^{n}(Q)}$ and $\mathcal{S}_{\mathcal{V}^{n}(Q)} * \mathcal{I}$ are invariant under translations of $\mathbb{R}^{n}$.

Proof. (i) The family $\mathcal{S}_{\mathcal{V}^{n}(Q)}$ is a semigroup of sets by Proposition 3.1. From Proposition 3.2 and Corollary 3.1, the families $\mathcal{S}_{\mathcal{V}^{n}(Q)} * \mathcal{I}$ and $\mathcal{I} * \mathcal{S}_{\mathcal{V}^{n}(Q)}$ are semigroups of sets. The inclusions are easily observed by Proposition 3.3.

(ii) (a) The result follows by applying Lemma 5.2 and Proposition 3.4: the families $\mathcal{A}$ and $\mathcal{B}$ in Proposition 3.4 are considered as the families $\mathcal{V}^{n}(Q)$ and $\mathcal{O}^{n}$ respectively, and Lemma 5.2 plays the same role as condition (b) in Proposition 3.4. The particular case follows from the inclusion $\mathcal{S}_{\mathcal{V}^{n}(Q)} \subset \mathcal{S}_{\mathcal{V}^{n}(Q)} * \mathcal{I}$ 
(b) Let $A \in \mathcal{S}_{\mathcal{V}^{n}(Q)} * \mathcal{I}$. Then $A=\left(U \backslash I_{1}\right) \cup I_{2}$ for some $U \in \mathcal{S}_{\mathcal{V}^{n}(Q)}$ and $I_{1}, I_{2} \in \mathcal{I}$. Since $\varnothing \neq A \subset \mathbb{R}^{n}$ then, we must have $0 \leq \operatorname{dim} A \leq n$.

Assume that $\operatorname{dim} A=n$. By the Brouwer-Dimension Theorem, there must exist a non-empty open set of $\mathbb{R}^{n}$ such that $O \subseteq A$. But $A=\left(U \backslash I_{1}\right) \cup I_{2} \subseteq$ $U \cup I_{2}$. By Lemma 5.2, there exists $V \in \mathcal{V}^{n}(Q)$ such that $V \subset O \backslash U$. So, $V \subset O \backslash U \subset\left(U \cup I_{2}\right) \backslash U \subseteq I_{2}$ and hence $V \in \mathcal{I}$ as $\mathcal{I}$ is an ideal of sets. This is in a contradiction with the condition $\mathcal{V}^{n}(Q) \cap \mathcal{I}=\varnothing$. So, $0 \leq \operatorname{dim} A \leq$ $n-1$. The particular case follows from the inclusion $\mathcal{S}_{\mathcal{V}^{n}(Q)} \subset \mathcal{S}_{\mathcal{V}^{n}(Q)} * \mathcal{I}$.

(iii) The invariance of the families $\mathcal{I} * \mathcal{S}_{\mathcal{V}^{n}(Q)}$ and $\mathcal{S}_{\mathcal{V}^{n}(Q)} * \mathcal{I}$ under translations of $\mathbb{R}^{n}$ follows from Proposition 5.2 and the assumption made on $\mathcal{I}$.

Question 5.1.1. What is the dimension of an element $U \in \mathcal{S}_{\mathcal{V}^{n}(Q)}$ ?

A partial answer on this question will be given by Proposition 5.8 in Section 5.3 of this chapter.

Let $\mathcal{M}^{n}$ be the $\sigma$-ideal of meager subsets of $\mathbb{R}^{n}$ and let $\mathcal{B}_{p}^{n}$ be the family of all subsets of $\mathbb{R}^{n}$ having the Baire property. Observe that $\mathcal{B}_{p}^{n}=\mathcal{O}^{n} * \mathcal{M}^{n}$ by Definition 2.10. Note that $\mathcal{M}^{n}$ is invariant under translations of $\mathbb{R}^{n}$ and from Proposition 5.1 (iii), we have $\mathcal{V}^{n}(Q) \cap \mathcal{M}^{n}=\varnothing$.

Corollary 5.1. The families $\mathcal{M}^{n} * \mathcal{S}_{\mathcal{V}^{n}(Q)}$ and $\mathcal{S}_{\mathcal{V}^{n}(Q)} * \mathcal{M}^{n}$ are semigroups of sets such that $\mathcal{S}_{\mathcal{V}^{n}(Q)} \subset \mathcal{M}^{n} * \mathcal{S}_{\mathcal{V}^{n}(Q)} \subset \mathcal{S}_{\mathcal{V}^{n}(Q)} * \mathcal{M}^{n}$. They are invariant under translations of $\mathbb{R}^{n}$, and consist of sets without the Baire property. In particular, the family $\mathcal{S}_{\mathcal{V}^{n}(Q)}$ consists of sets without the Baire property. Moreover, for each $A \in \mathcal{S}_{\mathcal{V}^{n}(Q)} * \mathcal{M}^{n}$ we have $0 \leq \operatorname{dim} A \leq n-1$.

Proof. From Proposition 5.3 item (ii), we have the equality $\left(\mathcal{S}_{\mathcal{V}^{n}(Q)} * \mathcal{M}^{n}\right) \cap\left(\mathcal{O}^{n} * \mathcal{M}^{n}\right)=\varnothing$. Keeping in mind that $\mathcal{B}_{p}^{n}=\mathcal{O}^{n} * \mathcal{M}^{n}$, it follows that $\mathcal{S}_{\mathcal{V}^{n}(Q)} * \mathcal{M}^{n} \subset \mathcal{P}\left(\mathbb{R}^{n}\right) \backslash \mathcal{B}_{p}^{n}$. In particular, from the inclusion $\mathcal{S}_{\mathcal{V}^{n}(Q)} \subset \mathcal{S}_{\mathcal{V}^{n}(Q)} * \mathcal{M}^{n}$, it follows that $\mathcal{S}_{\mathcal{V}^{n}(Q)} \subset \mathcal{P}\left(\mathbb{R}^{n}\right) \backslash \mathcal{B}_{p}^{n}$.

Let $\mathcal{I}_{f}$ (resp. $\mathcal{I}_{c}, \mathcal{I}_{c d}$ or $\mathcal{I}_{n}$ ) be the ideal of finite (resp. countable, closed and discrete or nowhere dense) subsets of $\mathbb{R}^{n}$. Like on the real line, for these ideals of sets, we have the inclusions $\mathcal{I}_{f} \subsetneq \mathcal{I}_{c d} \subsetneq \mathcal{I}_{c} \subsetneq \mathcal{M}^{n}$ and $\mathcal{I}_{c d} \subsetneq \mathcal{I}_{n} \subsetneq \mathcal{M}^{n}$ and they are invariant under translations of $\mathbb{R}^{n}$. 
Corollary 5.2. Let $\mathcal{I}$ be $\mathcal{I}_{f}, \mathcal{I}_{c}, \mathcal{I}_{c d}$ or $\mathcal{I}_{n}$. Then the families $\mathcal{I} * \mathcal{S}_{\mathcal{V}^{n}(Q)}$ and $\mathcal{S}_{\mathcal{V}^{n}(Q)} * \mathcal{I}$ are semigroups of sets such that $\mathcal{S}_{\mathcal{V}^{n}(Q)} \subset \mathcal{I} * \mathcal{S}_{\mathcal{V}^{n}(Q)} \subset \mathcal{S}_{\mathcal{V}^{n}(Q)} * \mathcal{I}$. They are invariant under translations of $\mathbb{R}^{n}$ and consist of sets without the Baire property having the dimension $\operatorname{dim} \leq n-1$.

Proof. The results follow from Proposition 5.3.

Remark 5.1. From the inclusions $\mathcal{I}_{f} \subsetneq \mathcal{I}_{c d} \subsetneq \mathcal{I}_{c} \subsetneq \mathcal{M}^{n}$ and $\mathcal{I}_{c d} \subsetneq \mathcal{I}_{n} \subsetneq \mathcal{M}^{n}$ it follows that

(a) $\mathcal{S}_{\mathcal{V}^{n}(Q)} * \mathcal{I}_{f} \subset \mathcal{S}_{\mathcal{V}^{n}(Q)} * \mathcal{I}_{c d} \subset \mathcal{S}_{\mathcal{V}^{n}(Q)} * \mathcal{I}_{c} \subset \mathcal{S}_{\mathcal{V}^{n}(Q)} * \mathcal{M}^{n}$ and $\mathcal{S}_{\mathcal{V}^{n}(Q)} * \mathcal{I}_{c d} \subset \mathcal{S}_{\mathcal{V}^{n}(Q)} * \mathcal{I}_{n} \subset \mathcal{S}_{\mathcal{V}^{n}(Q)} * \mathcal{M}^{n}$

(b) $\mathcal{I}_{f} * \mathcal{S}_{\mathcal{V}^{n}(Q)} \subset \mathcal{I}_{c d} * \mathcal{S}_{\mathcal{V}^{n}(Q)} \subset \mathcal{I}_{c} * \mathcal{S}_{\mathcal{V}^{n}(Q)} \subset \mathcal{M}^{n} * \mathcal{S}_{\mathcal{V}^{n}(Q)}$ and $\mathcal{I}_{c d} * \mathcal{S}_{\mathcal{V}^{n}(Q)} \subset \mathcal{I}_{n} * \mathcal{S}_{\mathcal{V}^{n}(Q)} \subset \mathcal{M}^{n} * \mathcal{S}_{\mathcal{V}^{n}(Q)}$.

It is interesting to know which of the semigroups coincide.

\subsection{Supersemigroup of Vitali selectors of $\mathbb{R}^{n}$}

Let $\mathcal{F}^{n}$ be the family of all countable, dense in the Euclidean topology, subgroups of $\left(\mathbb{R}^{n},+\right)$.

The following Propositions are valid and can be proved in similar ways as Proposition 4.7 and Proposition 4.8 on the real line.

Proposition 5.4. For each $Q_{1} \in \mathcal{F}^{n}$ there is a $Q_{2} \in \mathcal{F}^{n}$ such that $Q_{1} \subsetneq Q_{2}$ and $\left|Q_{2} / Q_{1}\right|=\aleph_{0}$.

Proposition 5.5. Let $Q_{1}, Q_{2}$ be elements of $\mathcal{F}^{n}$ such that $Q_{1} \subsetneq Q_{2}$ and $\left|Q_{2} / Q_{1}\right|=\aleph_{0}$. Then $\mathcal{S}_{\mathcal{V}^{n}\left(Q_{1}\right)} \cap \mathcal{S}_{\mathcal{V}^{n}\left(Q_{2}\right)}=\varnothing$.

Proposition 5.5 indicates that in the family of all semigroups of sets generated by Vitali selectors of $\mathbb{R}^{n}$, there is no element which contains all others.

Definition 5.2. Put $\mathcal{V}^{\text {sup }}=\left\{V: V \in \mathcal{V}(Q), Q \in \mathcal{F}^{n}\right\}$. The family $\mathcal{S}_{\mathcal{V}^{\text {sup }}}$ is called the supersemigroup of sets based on Vitali selectors of $\mathbb{R}^{n}$.

Like on the real line, proceeding in the same way as in Lemma 4.2, one can prove the following Lemma.

Lemma 5.3. For any set $U \in \mathcal{S}_{\mathcal{V}}$ sup and any non-empty open set $O$ of $\mathbb{R}^{n}$, there is a set $V \in \mathcal{V}^{\text {sup }}$ such that $V \subset O \backslash U$. 
Proposition 5.6. Let $\mathcal{I}$ be an ideal of subsets of $\mathbb{R}^{n}$. Then the following statements hold.

(i) The families $\mathcal{S}_{\mathcal{V} \text { sup }}, \mathcal{I} * \mathcal{S}_{\mathcal{V} \text { sup }}$ and $\mathcal{S}_{\mathcal{V} \text { sup }} * \mathcal{I}$ are semigroups of sets such that $\mathcal{S}_{\mathcal{V} \text { sup }} \subset \mathcal{I} * \mathcal{S}_{\mathcal{V} \text { sup }} \subset \mathcal{S}_{\mathcal{V} \text { sup }} * \mathcal{I}$.

(ii) If $\mathcal{V}^{\sup } \cap \mathcal{I}=\varnothing$ then

(a) $\left(\mathcal{S}_{\mathcal{V} \text { sup }} * \mathcal{I}\right) \cap\left(\mathcal{O}^{n} * \mathcal{I}=\varnothing\right)$.

In particular, $\mathcal{S}_{\mathcal{V}^{\text {sup }}} \cap\left(\mathcal{O}^{n} * \mathcal{I}\right)=\varnothing$.

(b) For each $A \in \mathcal{S}_{\mathcal{V}^{\text {sup }}} * \mathcal{I}$, we have $0 \leq \operatorname{dim} A \leq n-1$. In particular, for each

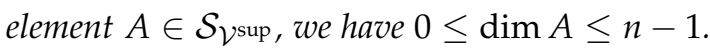

(iii) If $\mathcal{I}$ is invariant under translations of $\mathbb{R}^{n}$, then the families $\mathcal{I} * \mathcal{S}_{\mathcal{V} \text { sup }}$ and $\mathcal{S}_{\mathcal{V} \text { sup }} * \mathcal{I}$ are also invariant under translations of $\mathbb{R}^{n}$.

(iv) For each $Q \in \mathcal{F}^{n}$, we have $\mathcal{S}_{\mathcal{V}^{n}(Q)} * \mathcal{I} \subset \mathcal{S}_{\mathcal{V}^{\text {sup }}} * \mathcal{I}$.

Proof. (i) The family $\mathcal{S}_{\mathcal{V} \text { sup }}$ is a semigroup of sets by Proposition 3.1. By Proposition 3.2 and Corollary 3.1 the families $\mathcal{I} * \mathcal{S}_{\mathcal{V} \text { sup }}$ and $\mathcal{S}_{\mathcal{V} \text { sup }} * \mathcal{I}$ are semigroups of sets. The inclusions follow easily from Proposition 3.3.

(ii) (a) The equality $\left(\mathcal{S}_{\mathcal{V} \text { sup }} * \mathcal{I}\right) \cap\left(\mathcal{O}^{n} * \mathcal{I}=\varnothing\right)$ is proved by applying Proposition 3.4 and Lemma 5.3: the families $\mathcal{A}$ and $\mathcal{B}$ in Proposition 3.4 are considered as the families $\mathcal{V}^{\text {sup }}$ and $\mathcal{O}^{n}$, respectively, and Lemma 5.3 plays the same role as condition (b) in Proposition 3.4. The particular case follows from the inclusion $\mathcal{S}_{\mathcal{V} \text { sup }} \subset \mathcal{S}_{\mathcal{V} \text { sup }} * \mathcal{I}$.

(b) Let $A \in \mathcal{S}_{\mathcal{V} \text { sup }} * \mathcal{I}$. Then $A=(U \backslash M) \cup N$ where $U \in \mathcal{S}_{\mathcal{V}}$ sup and $M, N \in \mathcal{I}$. The equality $\mathcal{V}^{\text {sup }} \cap \mathcal{I}=\varnothing$ implies that $A \neq \varnothing$. Since $A \subset \mathbb{R}$, we must have $0 \leq \operatorname{dim} A \leq n$.

Assume that $\operatorname{dim} A=n$. By the Brouwer-Dimension Theorem, there must exist a non-empty open set $O$ in $\mathbb{R}^{n}$ such that $O \subseteq A$. But $A=(U \backslash M) \cup$ $N \subseteq U \cup N$. By Lemma 5.3, there exists $V \in \mathcal{V}^{\text {sup }}$ such that $V \subset O \backslash U$. So, $V \subset O \backslash U \subseteq(U \cup N) \backslash U \subseteq N$ which implies that $V$ is an element of $\mathcal{I}$. This is a contradiction. So $0 \leq \operatorname{dim} A \leq n-1$. The particular follows directly from the inclusion $\mathcal{S}_{\mathcal{V} \text { sup }} \subset \mathcal{S}_{\mathcal{V} \text { sup }} * \mathcal{I}$.

(iii) The statement is evident by Proposition 5.2 and the assumption on $\mathcal{I}$. 
(iv) The result follow from Proposition 5.5 and the definition of $\mathcal{S}_{\mathcal{V}}$ sup.

From Proposition 5.1, (iii), we have observed that $\mathcal{V}^{n}(Q) \cap \mathcal{M}^{n}=\varnothing$ for each $Q \in \mathcal{F}^{n}$. This observation together with the definition of $\mathcal{V}^{\text {sup }}$ imply that $\mathcal{V}^{\text {sup }} \cap \mathcal{M}^{n}=\varnothing$.

Corollary 5.3. The families $\mathcal{M}^{n} * \mathcal{S}_{\mathcal{V}}$ sup and $\mathcal{S}_{\mathcal{V}}$ sup $* \mathcal{M}^{n}$ are semigroups of sets such that $\mathcal{S}_{\mathcal{V} \text { sup }} \subset \mathcal{M}^{n} * \mathcal{S}_{\mathcal{V} \text { sup }} \subset \mathcal{S}_{\mathcal{V} \text { sup }} * \mathcal{M}^{n}$. They are invariant under translations of $\mathbb{R}^{n}$ and consist of sets without the Baire property. In particular, the family $\mathcal{S}_{\mathcal{V}}$ sup consists of sets without the Baire property. Moreover, for each element $A \in \mathcal{S}_{\mathcal{V}}$ sup $* \mathcal{M}^{n}$ we have $0 \leq \operatorname{dim} A \leq n-1$.

Proof. Note that by Proposition 5.6, we have $\left(\mathcal{S}_{\mathcal{V}^{\text {sup }}} * \mathcal{M}^{n}\right) \cap\left(\mathcal{O}^{n} * \mathcal{M}^{n}\right)=\varnothing$. Bearing in mind that $\mathcal{B}_{p}^{n}=\mathcal{O}^{n} * \mathcal{M}^{n}$, it follows that $\mathcal{S}_{\mathcal{V}^{\text {sup }}} * \mathcal{M}^{n} \subset \mathcal{P}\left(\mathbb{R}^{n}\right) \backslash \mathcal{B}_{p}^{n}$. In particular, from the inclusion $\mathcal{S}_{\mathcal{V}^{\text {sup }}} \subset \mathcal{S}_{\mathcal{V}^{\text {sup }}} * \mathcal{M}^{n}$, it follows that $\mathcal{S}_{\mathcal{V} \text { sup }} \subset \mathcal{P}(\mathbb{R}) \backslash \mathcal{B}_{p}^{n}$

The statement about dimension follows from Proposition 5.6, item (ii).

Corollary 5.4. Let $\mathcal{I}$ be $\mathcal{I}_{f}, \mathcal{I}_{c}, \mathcal{I}_{c d}$ or $\mathcal{I}_{n}$. Then the families $\mathcal{I} * \mathcal{S}_{\mathcal{V}}$ sup and $\mathcal{S}_{\mathcal{V}}$ sup $* \mathcal{I}$ are semigroups of sets such that $\mathcal{S}_{\mathcal{V}}$ sup $\subset \mathcal{I} * \mathcal{S}_{\mathcal{V}}$ sup $\subset \mathcal{S}_{\mathcal{V}}$ sup $* \mathcal{I}$. They are invariant under translations of $\mathbb{R}^{n}$ and consist of sets without the Baire property having the dimension $\operatorname{dim} \leq n-1$.

Proof. The results follow from Proposition 5.6.

\subsection{Rectangular Vitali selectors of $\mathbb{R}^{n}$}

Let $n \geq 2$. For each $j \leq n$, choose an element $Q_{j} \in \mathcal{F}$. (Let us recall that $\mathcal{F}$ is the family of all countable, dense in the real line subgroups of the additive group $(\mathbb{R},+)$.)

Put $Q=\prod_{j=1}^{n} Q_{j} \subset \mathbb{R}^{n}$. As the set $Q$ is a finite product of dense subsets of $\mathbb{R}$, the set $Q$ is dense in the Euclidean space $\mathbb{R}^{n}$. Being a finite product of countable subgroups of $(\mathbb{R},+)$, the set $Q$ is a countable subgroup of $\left(\mathbb{R}^{n},+\right)$. Thus, $Q \in \mathcal{F}^{n}$.

Definition 5.3. An element $Q \in \mathcal{F}^{n}$ is called rectangular if $Q=\prod_{j=1}^{n} Q_{j}$ where $Q_{j} \in \mathcal{F}$ for each $j=1,2, \ldots, n$.

Remark 5.2. There are elements of $\mathcal{F}^{n}$ which are not rectangular. 


\section{Example 5.1}

Let $\mathbb{D}=\left\{(x, y) \in \mathbb{R}^{2}: x-y \in \mathbb{Q}\right.$ and $\left.x \in \sqrt{2} \mathbf{Q}\right\}$. It is clear that $\mathbb{D}$ is a countable subgroup of $\left(\mathbb{R}^{2},+\right)$ and it can be written as $\mathbb{D}=\{(\sqrt{2} b, a+\sqrt{2} b): a, b \in \mathbb{Q}\}$. The set $\mathbb{D}$ is also dense in $\mathbb{R}^{2}$.

Let $\operatorname{Proj}_{1}(x, y)=x, \operatorname{Proj}_{2}(x, y)=y$. Note that $\operatorname{Proj}_{1} \mathbb{D}=\sqrt{2} \mathbf{Q}$ and $\operatorname{Proj}_{2} \mathbb{D}=\mathbb{Q}(\sqrt{2})$, where $\mathbb{Q}(\sqrt{2})=\{a+b \sqrt{2}: a, b \in \mathbb{Q}\}$.

Assume that $\mathbb{D}$ is a rectangular subgroup of $\left(\mathbb{R}^{2},+\right)$. So $\mathbb{D}=\sqrt{2} \mathbf{Q} \times \mathbb{Q}(\sqrt{2})$. But $(\sqrt{2}, 2 \sqrt{2}) \notin \mathbb{D}$ and $(\sqrt{2}, 2 \sqrt{2}) \in \sqrt{2} \mathbb{Q} \times \mathbb{Q}(\sqrt{2})$. We have a contradiction. Thus, $\mathbb{D}$ is not a rectangular subgroup of $\left(\mathbb{R}^{2},+\right)$.

Definition 5.4. Let $Q=\prod_{j=1}^{n} Q_{j}$ where $Q_{j} \in \mathcal{F}$ for each $j=1,2, \ldots, n$. A rectangular Vitali $Q$ - selector of $\mathbb{R}^{n}$ is any subset $\bar{V}$ of $\mathbb{R}^{n}$ such that $\bar{V}=\prod_{j=1}^{n} V(j)$, where $V(j) \in \mathcal{V}\left(Q_{j}\right)$ for $j=1,2, \cdots, n$.

The family of all rectangular Vitali selectors associated to a rectangular subgroup $Q$ will be denoted by $r \mathcal{V}^{n}(Q)$. The notation $\mathcal{S}_{r \mathcal{V}^{n}(Q)}$ will be used for the semigroup of sets generated by the family $r \mathcal{V}^{n}(Q)$.

Proposition 5.7. Let $\bar{V}$ be a rectangular Vitali $Q$-selector of $\mathbb{R}^{n}$. Then, the set $\bar{V}$ is a Vitali $Q$-selector of $\mathbb{R}^{n}$.

Proof. Let $\bar{V}$ be a rectangular Vitali $Q$-selector related to a rectangular subgroup $Q$. Since $Q$ is a rectangular subgroup, then $Q=\prod_{j=1}^{n} Q_{j}$, where $Q_{j} \in \mathcal{F}$ for each $j \leq n$.

For an element $\bar{x}=\left(x_{1}, \cdots, x_{n}\right) \in \mathbb{R}^{n}$, its equivalence class is given by $Q_{\bar{x}}=$ $\prod_{j=1}^{n}\left(Q_{j}\right)_{x_{j}}$. Since $\bar{V}$ is a rectangular Vitali $Q$-selector of $\mathbb{R}^{n}$, then $\bar{V}=\prod_{j=1}^{n} V(j)$ where $V(j) \in \mathcal{V}\left(Q_{j}\right)$. It follows that $Q_{\bar{x}} \cap \bar{V}=\left(\prod_{j=1}^{n}\left(Q_{j}\right)_{x_{j}}\right) \cap\left(\prod_{j=1}^{n} V(j)\right)=$ $\prod_{j=1}^{n}\left(\left(Q_{j}\right)_{x_{j}} \cap V(j)\right)$. Since each $V(j)$ is a Vitali $Q_{j}-$ selector of $\mathbb{R}$, then we have $\left|\left(Q_{j}\right)_{x_{j}} \cap V(j)\right|=1$ for each $j \leq n$. It follows that the intersection $Q_{x} \cap \bar{V}$ consists of only one element of $\mathbb{R}^{n}$. So, the set $\bar{V}$ is a Vitali $Q$-selector of $\mathbb{R}^{n}$.

By Proposition 5.7, we have the inclusion $r \mathcal{V}^{n}(Q) \subset \mathcal{V}^{n}(Q)$ for each rectangular subgroup $Q$ of $\mathbb{R}^{n}, n \geq 2$. Note that for $n=1$, we have the equality $r \mathcal{V}(Q)=\mathcal{V}(Q)$.

As we will see in the following example, for a rectangular subgroup $Q$, there are Vitali $Q$-selectors of $\mathbb{R}^{n}$ for $n \geq 2$, which are not rectangular Vitali $Q$-selectors. So, $r \mathcal{V}^{n}(Q) \neq \mathcal{V}^{n}(Q)$ in this case. 


\section{Example 5.2}

Let $Q=\mathbb{Q} \times \mathbb{Q}$, where $\mathbb{Q}$ is the set of rational numbers. Consider a Vitali $Q$-selector $V$ of $\mathbb{R}^{2}$ containing the points $(1,1)$ and $(2, \sqrt{2})$. The set $V$ can not be a rectangular Vitali $Q$-selector of $\mathbb{R}^{2}$.

For, if $V$ is a rectangular Vitali $Q$-selector of $\mathbb{R}^{2}$, then $V=V_{1} \times V_{2}$ where $V_{j}$ is a Vitali $Q$-selector of $\mathbb{R}$ for $j=1,2$. Since $V_{1}$ could not contain the points 1 and 2, we have a contradiction. This shows that $V$ can not be a rectangular Vitali $Q$-selector of $\mathbb{R}^{2}$.

Lemma 5.4. The families $r \mathcal{V}^{n}(Q)$ and $\mathcal{S}_{r \mathcal{V}^{n}(Q)}$ are invariant under translations of $\mathbb{R}^{n}$.

Proof. Let $\bar{V}=\prod_{j=1}^{n} V(j)$ where $V(j) \in \mathcal{V}\left(Q_{j}\right), j \leq n$ and let $\bar{x}=\left(x_{1}, \cdots, x_{n}\right) \in$ $\mathbb{R}^{n}$ where each $x_{j} \in \mathbb{R}, j \leq n$. Note that $\bar{V}_{\bar{x}}=\prod_{j=1}^{n}(V(j))_{x_{j}}$. By Lemma 4.1, the set $(V(j))_{x_{j}}$ is a Vitali $Q_{j}$-selector of $\mathbb{R}$ for each $j \leq n$. This shows that $\bar{V}$ is a finite product of Vitali $Q_{j}$-selectors of $\mathbb{R}$. Hence, the set $\bar{V}_{\bar{x}}$ is a rectangular Vitali $Q$-selector of $\mathbb{R}^{n}$.

Since the family $\mathcal{S}_{r \mathcal{V}^{n}(Q)}$ consists of finite unions of elements in $r \mathcal{V}^{n}(Q)$, then it is also invariant under translations of $\mathbb{R}^{n}$.

Proposition 5.8. For each element $U$ of $\mathcal{S}_{r \mathcal{V}^{n}(Q)}$ there are elements $U(j) \in \mathcal{S}_{\mathcal{V}\left(Q_{j}\right)}, j \leq$ $n$, such that $U \subseteq \prod_{j=1}^{n} U(j)$. Moreover $\prod_{j=1}^{n} U(j) \in \mathcal{S}_{r \mathcal{V}^{n}(Q)}$ and $\operatorname{dim}\left(\prod_{j=1}^{n} U(j)\right)=$ $\operatorname{dim} U=0$.

Proof. Let $U \in \mathcal{S}_{r \mathcal{V}^{n}(Q)}$. Then $U=\bigcup_{i=1}^{m} \bar{V}(i)$, where $\bar{V}(i) \in r \mathcal{V}^{n}(Q)$ for each $i \leq m$. Thereby $\bar{V}(i)=\prod_{j=1}^{n} V(i, j)$ where $V(i, j) \in \mathcal{V}\left(Q_{j}\right)$ for each $i \leq m$ and $j \leq n$.

For each $j \leq n$ put $U(j)=\bigcup_{i=1}^{m} V(i, j)$ and note that $U(j) \in \mathcal{S}_{\mathcal{V}\left(Q_{j}\right)}$. Let us also note that $U \subseteq \prod_{j=1}^{n} U(j)=\prod_{j=1}^{n}\left(\bigcup_{i=1}^{m} V(i, j)\right)=\bigcup\left\{\prod_{j=1}^{n} V\left(i_{j}, j\right)\right.$ : $\left.\left(i_{1}, \cdots, i_{n}\right) \in\{1, \cdots, m\}^{n}\right\} \in \mathcal{S}_{r \mathcal{V}^{n}(Q)}$. Since $\operatorname{dim} U(j)=0$ for each $j \leq n$ (by Proposition 4.4), we get $\operatorname{dim}\left(\prod_{j=1}^{n} U(j)\right)=0$ (see the Product Theorem for dimension). Additionally, by the Monotonicity property of dimension, we have $\operatorname{dim} U=0$.

Lemma 5.5. For any set $U \in \mathcal{S}_{r \mathcal{V}^{n}(Q)}$ and any non-empty open set $O$ of $\mathbb{R}^{n}$ there is a set $\bar{V} \in r \mathcal{V}^{n}(Q)$ such that $\bar{V} \subset O \backslash U$. 
Proof. Let $U \in \mathcal{S}_{r \mathcal{V}^{n}(Q)}$ and let $O$ be a non-empty open set of $\mathbb{R}^{n}$. Then $U=$ $\bigcup_{i=1}^{m} \bar{V}(i)$, where $\bar{V}(i) \in r \mathcal{V}^{n}(Q)$ for each $i \leq m$.

By Proposition 5.8 there are elements $U(j) \in \mathcal{S}_{\mathcal{V}\left(Q_{j}\right)}, j \leq n$ such that $U \subseteq$ $\prod_{j=1}^{n} U(j)$. By the definition of the product topology on $\mathbb{R}^{n}$ there are non-empty open sets $O_{j}, j \leq n$ of $\mathbb{R}$ such that $\prod_{j=1}^{n} O_{j} \subseteq O$. In addition, by Lemma 4.2, for each $j \leq n$ there is a Vitali $Q_{j}$-selector $V_{j}$ of $\mathbb{R}$ such that $V_{j} \subset O_{j} \backslash U(j)$. Let us notice that $O \backslash U \supset \prod_{j=1}^{n} O_{j} \backslash \prod_{j=1}^{n} U(j) \supset \prod_{j=1}^{n}\left(O_{j} \backslash U(j)\right) \supset \prod_{j=1}^{n} V_{j}$ and $\bar{V}=\prod_{j=1}^{n} V_{j} \in r \mathcal{V}^{n}(Q)$.

Recall that $\mathcal{O}^{n}$ is the family of all open subsets of $\mathbb{R}^{n}$ and $\mathcal{O}^{n}$ is a semigroup of subsets of $\mathbb{R}^{n}$ satisfying the equality $\mathcal{S}_{\mathcal{O}^{n}}=\mathcal{O}^{n}$.

Proposition 5.9. Let $\mathcal{I}$ be an ideal of subsets of $\mathbb{R}^{n}$. Then the following statements hold.

(i) The families $\mathcal{S}_{r \mathcal{V}^{n}(Q)}, \mathcal{I} * \mathcal{S}_{r \mathcal{V}^{n}(Q)}$ and $\mathcal{S}_{r \mathcal{V}^{n}(Q)} * \mathcal{I}$ are semigroups of sets such that $\mathcal{S}_{r \mathcal{V}^{n}(Q)} \subset \mathcal{I} * \mathcal{S}_{r \mathcal{V}^{n}(Q)} \subset \mathcal{S}_{r \mathcal{V}^{n}(Q)} * \mathcal{I}$.

(ii) If $r \mathcal{V}^{n}(Q) \cap \mathcal{I}=\varnothing$ then

(a) $\left(\mathcal{S}_{r \mathcal{V}^{n}(Q)} * \mathcal{I}\right) \cap\left(\mathcal{O}^{n} * \mathcal{I}\right)=\varnothing$.

In particular, $\mathcal{S}_{r \mathcal{V}^{n}(Q)} \cap\left(\mathcal{O}^{n} * \mathcal{I}\right)=\varnothing$.

(b) For each element $A \in \mathcal{S}_{r \mathcal{V}^{n}(Q)} * \mathcal{I}$, we have $0 \leq \operatorname{dim} A \leq n-1$.

(iii) If $\mathcal{I}$ is invariant under translations of $\mathbb{R}^{n}$, then $\mathcal{I} * \mathcal{S}_{r \mathcal{V}^{n}(Q)}$ and $\mathcal{S}_{r \mathcal{V}^{n}(Q)} * \mathcal{I}$ are also invariant under translations of $\mathbb{R}^{n}$.

Proof. The proof of the statement is very close to the proof of Proposition 5.3 but for the reader convenience we suggest it too.

(i) The family $\mathcal{S}_{r \mathcal{V}^{n}(Q)}$ is a semigroup of sets by Proposition 3.1. By Proposition 3.2 and Corollary 3.1 the families $\mathcal{S}_{r \mathcal{V}^{n}(Q)} * \mathcal{I}$ and $\mathcal{I} * \mathcal{S}_{r \mathcal{V}^{n}(Q)}$ are semigroups of sets. The inclusion follows from Proposition 3.3.

(ii) (a) To prove the equality $\left(\mathcal{S}_{r \mathcal{V}^{n}(Q)} * \mathcal{I}\right) \cap\left(\mathcal{O}^{n} * \mathcal{I}\right)=\varnothing$, we apply Proposition 3.4 and Lemma 5.5, where the families $\mathcal{A}$ and $\mathcal{B}$ in Proposition 3.4 are considered as $r \mathcal{V}^{n}(Q)$ and $\mathcal{O}^{n}$ respectively. The particular case easily follows. 
(b) Let $A \in \mathcal{S}_{r \mathcal{V}^{n}(Q)} * \mathcal{I}$. Then $A=\left(U \backslash I_{1}\right) \cup I_{2}$ where $U \in \mathcal{S}_{r \mathcal{V}^{n}(Q)}$ and $I_{1}, I_{2} \in \mathcal{I}$. The condition $r \mathcal{V}^{n}(Q) \cap \mathcal{I}=\varnothing$ implies that $A \neq \varnothing$. Since $\varnothing \neq A \subset \mathbb{R}^{n}$ then, we must have $0 \leq \operatorname{dim} A \leq n$.

Assume that $\operatorname{dim} A=n$. By the Brouwer-Dimension Theorem, there must exist a non-empty open set of $\mathbb{R}^{n}$ such that $O \subset A=\left(U \backslash I_{1}\right) \cup I_{2} \subseteq U \cup I_{2}$. By Lemma 5.5, there exists a rectangular Vitali $Q-$ selector $\bar{V} \in r \mathcal{V}^{n}(Q)$ such that $\bar{V} \subset O \backslash U$. So, $\bar{V} \subset O \backslash U \subset\left(U \cup I_{2}\right) \backslash U \subset I_{2}$ and hence $\bar{V} \in \mathcal{I}$ as $\mathcal{I}$ is an ideal of sets. This is a contradiction because $r \mathcal{V}^{n}(Q) \cap \mathcal{I}=\varnothing$. So, $0 \leq \operatorname{dim} A \leq n-1$.

(iii) By Lemma 5.4 and the assumption made on $\mathcal{I}$, the statement is evident.

Recall that $\mathcal{M}^{n}$ is the $\sigma$-ideal of meager sets in $\mathbb{R}^{n}$ and $\mathcal{B}_{p}^{n}$ is the family of all subsets of $\mathbb{R}^{n}$ having the Baire property in $\mathbb{R}^{n}$. We have observed that for each rectangular subgroup $Q$ of $\mathbb{R}^{n}$ the inclusion $r \mathcal{V}^{n}(Q) \subsetneq \mathcal{V}^{n}(Q)$ holds. By Proposition 5.1 (iii), we know that $\mathcal{V}^{n}(Q) \cap \mathcal{M}^{n}=\varnothing$. It follows that $r \mathcal{V}^{n}(Q) \cap \mathcal{M}^{n}=\varnothing$.

Corollary 5.5. The families $\mathcal{M}^{n} * \mathcal{S}_{r \mathcal{V}^{n}(Q)}$ and $\mathcal{S}_{r \mathcal{V}^{n}(Q)} * \mathcal{M}^{n}$ are semigroups of sets such that $\mathcal{S}_{r \mathcal{V}^{n}(Q)} \subset \mathcal{M}^{n} * \mathcal{S}_{r \mathcal{V}^{n}(Q)} \subset \mathcal{S}_{r \mathcal{V}^{n}(Q)} * \mathcal{M}^{n}$. They are invariant under translations of $\mathbb{R}^{n}$, and consist of sets without the Baire property. In particular, the family $\mathcal{S}_{r \mathcal{V}^{n}(Q)}$ consists of sets without the Baire property. Moreover, for each element $A$ of $\mathcal{S}_{r \mathcal{V}^{n}(Q)} * \mathcal{M}^{n}$, we have $0 \leq \operatorname{dim} A \leq n-1$.

Proof. By Proposition 5.9, the equality $\left(\mathcal{S}_{r \mathcal{V}^{n}}(Q) * \mathcal{M}^{n}\right) \cap\left(\mathcal{O}^{n} * \mathcal{M}^{n}\right)=\varnothing$ holds. From the definition of sets with the Baire property, we know that $\mathcal{B}_{p}^{n}=\mathcal{O}^{n} * \mathcal{M}^{n}$. Thus $\mathcal{S}_{r \mathcal{V}^{n}(Q)} * \mathcal{M}^{n} \subset \mathcal{P}\left(\mathbb{R}^{n}\right) \backslash \mathcal{B}_{p}^{n}$. The particular case follows from the inclusion $\mathcal{S}_{r \mathcal{V}^{n}(Q)} \subset \mathcal{S}_{r \mathcal{V}^{n}(Q)} * \mathcal{M}^{n}$

Let $A \in \mathcal{S}_{r \mathcal{V}^{n}(Q)} * \mathcal{M}^{n}$. Taking the ideal $\mathcal{I}$ to be the family of all meager subsets of $\mathbb{R}^{n}$, from Proposition 5.9, we get $0 \leq \operatorname{dim} A \leq n-1$.

Corollary 5.6. Let $\mathcal{I}$ be $\mathcal{I}_{f}, \mathcal{I}_{c}, \mathcal{I}_{c d}$ or $\mathcal{I}_{n}$. Then the families $\mathcal{I} * \mathcal{S}_{r \mathcal{V}^{n}(Q)}$ and $\mathcal{S}_{r \mathcal{V}^{n}(Q)} * \mathcal{I}$ are semigroups of sets such that $\mathcal{S}_{r \mathcal{V}^{n}(Q)} \subset \mathcal{I} * \mathcal{S}_{r \mathcal{V}^{n}(Q)} \subset \mathcal{S}_{r \mathcal{V}^{n}(Q)} * \mathcal{I}$. They are invariant under translations of $\mathbb{R}^{n}$, and consist of sets without the Baire property having the dimension $\operatorname{dim} \leq n-1$.

Proof. Apply Proposition 5.9. 
Remark 5.3. From the inclusions $\mathcal{I}_{f} \subsetneq \mathcal{I}_{c d} \subsetneq \mathcal{I}_{c} \subsetneq \mathcal{M}^{n}$ and $\mathcal{I}_{c d} \subsetneq \mathcal{I}_{n} \subsetneq \mathcal{M}^{n}$ it follows that

(a) $\mathcal{S}_{r \mathcal{V}^{n}(Q)} * \mathcal{I}_{f} \subset \mathcal{S}_{r \mathcal{V}^{n}(Q)} * \mathcal{I}_{c d} \subset \mathcal{S}_{r \mathcal{V}^{n}(Q)} * \mathcal{I}_{c} \subset \mathcal{S}_{r \mathcal{V}^{n}(Q)} * \mathcal{M}^{n}$ and $\mathcal{S}_{r \mathcal{V}^{n}(Q)} * \mathcal{I}_{c d} \subset \mathcal{S}_{r \mathcal{V}^{n}(Q)} * \mathcal{I}_{n} \subset \mathcal{S}_{r \mathcal{V}^{n}(Q)} * \mathcal{M}^{n}$

(b) $\mathcal{I}_{f} * \mathcal{S}_{r \mathcal{V}^{n}(Q)} \subset \mathcal{I}_{c d} * \mathcal{S}_{r \mathcal{V}^{n}(Q)} \subset \mathcal{I}_{c} * \mathcal{S}_{r \mathcal{V}^{n}(Q)} \subset \mathcal{M}^{n} * \mathcal{S}_{r \mathcal{V}^{n}(Q)}$ and $\mathcal{I}_{c d} * \mathcal{S}_{r \mathcal{V}^{n}(Q)} \subset \mathcal{I}_{n} * \mathcal{S}_{r \mathcal{V}^{n}(Q)} \subset \mathcal{M}^{n} * \mathcal{S}_{r \mathcal{V}^{n}(Q)}$.

It is interesting to know which of the semigroups coincide.

\subsubsection{Supersemigroup of rectangular Vitali selectors of $\mathbb{R}^{n}$}

Let $r \mathcal{F}^{n}$ be the family of all rectangular subgroups of $\left(\mathbb{R}^{n},+\right)$.

Proposition 5.10. For each element $Q^{1} \in r \mathcal{F}^{n}$ there is an element $Q^{2} \in r \mathcal{F}^{n}$ such that $r \mathcal{V}^{n}\left(Q^{1}\right) \cap r \mathcal{V}^{n}\left(Q^{2}\right)=\varnothing$ and $\mathcal{S}_{r \mathcal{V}^{n}\left(Q^{1}\right)} \cap \mathcal{S}_{r \mathcal{V}^{n}\left(Q^{2}\right)}=\varnothing$.

Proof. Let $Q^{1}=\prod_{j=1}^{n} Q_{j}^{1}$, where $Q_{j}^{1} \in \mathcal{F}$ for all $j \leq n$. Consider an element $Q \in \mathcal{F}$ such that $Q_{1}^{1} \subsetneq Q$ and $\left|Q / Q_{1}^{1}\right|=\aleph_{0}$. This can be done by Proposition 4.7.

Put $Q^{2}=\prod_{j=1}^{n} Q_{j}^{2}$, where $Q_{1}^{2}=Q$ and $Q_{j}^{2}=Q_{j}^{1}$ for all $j \geq 2$. It is clear that $Q^{2} \in r \mathcal{F}^{n}$.

Since $Q_{1}^{1} \subsetneq Q_{1}^{2}$ then $Q^{1} \subsetneq Q^{2}$. By Proposition 4.6, we know that $\mathcal{V}\left(Q_{1}^{1}\right) \cap \mathcal{V}\left(Q_{1}^{2}\right)=\varnothing$. This implies that $r \mathcal{V}^{n}\left(Q^{1}\right) \cap r \mathcal{V}^{n}\left(Q^{2}\right)=\varnothing$.

Assume there exists $U \in \mathcal{S}_{r \mathcal{V}^{n}\left(Q^{1}\right)} \cap \mathcal{S}_{r \mathcal{V}^{n}\left(Q^{2}\right)}$. Since $U \in \mathcal{S}_{r \mathcal{V}^{n}\left(Q^{2}\right)}$, then by Proposition 5.8, there are elements $U(j) \in \mathcal{S}_{\mathcal{V}\left(Q_{j}^{2}\right)}, j \leq n$, such that $U \subseteq \prod_{j=1}^{n} U(j)$.

Let $E_{\alpha}\left(Q_{1}^{2}\right)$ be an equivalence class in $\mathbb{R}$ with respect to $Q_{1}^{2}$. We have

$$
\left|U(1) \cap E_{\alpha}\left(Q_{1}^{2}\right)\right|<\aleph_{0}
$$

Since $\left|Q_{1}^{2} / Q_{1}^{1}\right|=\aleph_{0}$, we have $E_{\alpha}\left(Q_{1}^{2}\right)=\bigcup_{\beta \in J} E_{\beta}\left(Q_{1}^{1}\right)$ where $E_{\beta}\left(Q_{1}^{1}\right)$ are distinct equivalence classes with respect to $Q_{1}^{1}$ and $|J|=\aleph_{0}$.

As $U \in \mathcal{S}_{r \mathcal{V}^{n}\left(Q^{1}\right)}$, there are elements $V(j) \in \mathcal{V}\left(Q_{j}^{1}\right), j \leq n$, such that $\prod_{j=1}^{n} V(j) \subset$ $U$. It follows that $\prod_{j=1}^{n} V(j) \subset U \subseteq \prod_{j=1}^{n} U(j)$ and hence $V(1) \subset U(1)$. Note that $\left|U(1) \cap E_{\alpha}\left(Q_{1}^{2}\right)\right| \geq\left|V(1) \cap E_{\alpha}\left(Q_{1}^{2}\right)\right|=\left|V(1) \cap\left(\bigcup_{\beta \in J} E_{\beta}\left(Q_{1}^{1}\right)\right)\right|=\aleph_{0}$.

This is a contradiction. 
Proposition 5.10 indicates that in the family $r \mathcal{F}^{n}$ of all rectangular subgroups of $\mathbb{R}^{n}$, there is no element $Q$ such that the corresponding generated semigroup $\mathcal{S}_{r \mathcal{V}^{n}(Q)}$ contains all others.

Put $r \mathcal{V}_{\text {sup }}^{n}=\left\{\bar{V}: \bar{V} \in r \mathcal{V}^{n}(Q), Q \in r \mathcal{F}^{n}\right\}$ and let $\mathcal{S}_{r \mathcal{V}_{\text {sup }}^{n}}$ be the semigroup of sets generated by $r \mathcal{V}_{\text {sup }}^{n}$.

Definition 5.5. The family $\mathcal{S}_{r \mathcal{V}_{\text {sup }}^{n}}$ is called the supersemigroup of sets based on rectangular Vitali selectors of $\mathbb{R}^{n}$.

Proposition 5.11. The following statements hold.

(i) For each $Q \in r \mathcal{F}^{n}$ we have $r \mathcal{V}^{n}(Q) \subsetneq r \mathcal{V}_{\text {sup }}^{n}$ and $\mathcal{S}_{r \mathcal{V}^{n}(Q)} \subsetneq \mathcal{S}_{r \mathcal{V}_{\text {sup }}^{n}}$.

(ii) The families $r \mathcal{V}_{\text {sup }}^{n}$ and $\mathcal{S}_{r \mathcal{V}_{\text {sup }}^{n}}$ are invariant under translations of $\mathbb{R}^{n}$.

Proof. (i) From the definition of $r \mathcal{V}_{\text {sup }}^{n}$, we have the inclusions $r \mathcal{V}^{n}(Q) \subset r \mathcal{V}_{\text {sup }}^{n}$ and $\mathcal{S}_{r \mathcal{V}^{n}\left(Q^{n}\right)} \subset \mathcal{S}_{r \mathcal{V}_{\text {sup }}^{n}}$ for each $Q \in r \mathcal{F}^{n}$. The inequalities $r \mathcal{V}^{n}(Q) \neq r \mathcal{V}_{\text {sup }}^{n}$ and $\mathcal{S}_{r \mathcal{V}^{n}(Q)} \neq \mathcal{S}_{r \mathcal{V}_{\text {sup }}^{n}}$ follow from Proposition 5.10.

(ii) From the definition of $r \mathcal{V}_{\text {sup }}^{n}$, the set $r \mathcal{V}_{\text {sup }}^{n}$ can be written as follows:

$r \mathcal{V}_{\text {sup }}^{n}=\bigcup\left\{r \mathcal{V}^{n}(Q): Q \in r \mathcal{F}^{n}\right\}$. By Lemma 5.4, for each $Q \in r \mathcal{F}^{n}$ the family $r \mathcal{V}^{n}(Q)$ is invariant under translations of $\mathbb{R}^{n}$. Thus, the family $r \mathcal{V}_{\text {sup }}^{n}$ is a union of families which are invariant under translations of $\mathbb{R}^{n}$. So, the family $r \mathcal{V}_{\text {sup }}^{n}$ is invariant under translations of $\mathbb{R}^{n}$.

The family $\mathcal{S}_{r \mathcal{V}_{\text {sup }}^{n}}$ consists of all finite union of elements in $r \mathcal{V}_{\text {sup }}^{n}$. Thus, it is also invariant under translations of $\mathbb{R}^{n}$.

Lemma 5.6. For any set $U \in \mathcal{S}_{r \mathcal{V}_{\text {sup }}^{n}}$ and any non-empty open set $O$ of $\mathbb{R}^{n}$ there is a set $\bar{V} \in r \mathcal{V}_{\text {sup }}^{n}$ such that $\bar{V} \subset O \backslash U$.

Proof. Let $U \in \mathcal{S}_{r V_{\text {sup }}^{n}}$ and let $O$ be a non-empty open set of $\mathbb{R}^{n}$. By Proposition

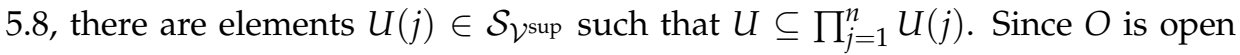
in the product topology of $\mathbb{R}^{n}$, there are non-empty open subsets $O_{j}$ of $\mathbb{R}$ such that $\prod_{j=1}^{n} O_{j} \subset O$.

By Lemma 4.3, we can find $V_{j} \in \mathcal{V}^{\text {sup }}$ such that $V_{j} \in O_{j} \backslash U(j)$ for each $j \leq n$.

Put $\bar{V}=\prod_{j=1}^{n} V_{j}$. The set $\bar{V}$ is a rectangular Vitali selector. Note that $\bar{V}=$ $\prod_{j=1}^{n} V_{j} \subset \prod_{j=1}^{n}\left(O_{j} \backslash U(j)\right) \subset \prod_{j=1}^{n} O_{j} \backslash \prod_{j=1}^{n} U(j) \subset O \backslash U$ and $\bar{V} \in r \mathcal{V}_{\text {sup }}^{n}$. 
Proposition 5.12. Let $\mathcal{I}$ be an ideal of subsets of $\mathbb{R}^{n}$. Then the following statements hold.

(i) The families $\mathcal{S}_{r \mathcal{V}_{\text {sup }}^{n}}, \mathcal{I} * \mathcal{S}_{r \mathcal{V}_{\text {sup }}^{n}}$ and $\mathcal{S}_{r \mathcal{V}_{\text {sup }}^{n}} * \mathcal{I}$ are semigroups of sets such that $\mathcal{S}_{r \mathcal{V}_{\text {sup }}^{n}} \subset \mathcal{I} * \mathcal{S}_{r \mathcal{V}_{\text {sup }}^{n}} \subset \mathcal{S}_{r \mathcal{V}_{\text {sup }}^{n}} * \mathcal{I}$.

(ii) If $r \mathcal{V}_{\text {sup }}^{n} \cap \mathcal{I}=\varnothing$ then

(a) $\left(\mathcal{S}_{r} \nu_{\text {sup }}^{n} * \mathcal{I}\right) \cap\left(\mathcal{O}^{n} * \mathcal{I}\right)=\varnothing$.

In particular, $\mathcal{S}_{r \mathcal{V}_{\text {sup }}^{n}} \cap\left(\mathcal{O}^{n} * \mathcal{I}\right)=\varnothing$.

(b) For each element $A$ of $\mathcal{S}_{\text {r } \mathcal{V}_{\text {sup }}^{n}} * \mathcal{I}$ we have $0 \leq \operatorname{dim} A \leq n-1$. In particular, for each element $A$ of $\mathcal{S}_{r} \mathcal{v}_{\text {sup }}^{n}$ we have $0 \leq \operatorname{dim} A \leq n-1$.

(iii) If $\mathcal{I}$ is invariant under translations of $\mathbb{R}^{n}$, then $\mathcal{I} * \mathcal{S}_{r \mathcal{V}_{\text {sup }}^{n}}$ and $\mathcal{S}_{r \mathcal{V}_{\text {sup }}^{n}} * \mathcal{I}$ are also invariant under translations of $\mathbb{R}^{n}$.

(iv) For each $Q \in r \mathcal{F}^{n}$ we have $\mathcal{S}_{r \mathcal{V}^{n}} * \mathcal{I} \subset \mathcal{S}_{r \mathcal{V}_{\text {sup }}^{n}} * \mathcal{I}$.

Proof. The proof of the statement is very close to the proof of Proposition 5.6 but for the reader convenience we suggest it too.

(i) The family $\mathcal{S}_{r} \nu_{\text {sup }}^{n}$ is a semigroup of sets by Proposition 3.1. By Proposition 3.2 and Corollary 3.1 the families $\mathcal{S}_{r} \mathcal{V}_{\text {sup }}^{n} * \mathcal{I}$ and $\mathcal{I} * \mathcal{S}_{r} \mathcal{V}_{\text {sup }}^{n}$ are semigroups of sets. The inclusion follows from Proposition 3.3.

(ii) (a) To prove the equality $\left(\mathcal{S}_{r} \mathcal{V}_{\text {sup }}^{n} * \mathcal{I}\right) \cap\left(\mathcal{O}^{n} * \mathcal{I}\right)=\varnothing$, we apply Proposition 3.4 and Lemma 5.6, where the families $\mathcal{A}$ and $\mathcal{B}$ in Proposition 3.4 are considered as the families $r \mathcal{V}_{\text {sup }}^{n}$ and $\mathcal{O}^{n}$, respectively. Lemma 5.6 plays the same role as condition (b) in Proposition 3.4. The particular case easily follows.

(b) Let $A \in \mathcal{S}_{r V_{\text {sup }}^{n}} * \mathcal{I}$. Then $A=\left(U \backslash I_{1}\right) \cup I_{2}$ where $U \in \mathcal{S}_{r \mathcal{V}_{\text {sup }}^{n}}$ and $I_{1}, I_{2} \in$ $\mathcal{I}$. The equality $r \mathcal{V}_{\text {sup }}^{n} \cap \mathcal{I}=\varnothing$ implies that $A \neq \varnothing$. Since $\varnothing \neq A \subset \mathbb{R}^{n}$ then, we must have $0 \leq \operatorname{dim} A \leq n$.

Assume that $\operatorname{dim} A=n$. By Brouwer-Dimension Theorem, there must exist a non-empty open set of $\mathbb{R}^{n}$ such that $O \subset A=\left(U \backslash I_{1}\right) \cup I_{2} \subseteq U \cup I_{2}$. By Lemma 5.6, there exists a rectangular Vitali $Q$-selector $\bar{V}$ in $r \mathcal{V}_{\text {sup }}^{n}$ such that $\bar{V} \subset O \backslash U$. So, $\bar{V} \subset O \backslash U \subset\left(U \cup I_{2}\right) \backslash U \subset I_{2}$ and hence $\bar{V} \in \mathcal{I}$ as $\mathcal{I}$ is an ideal of sets. This is a contradiction because $r \mathcal{V}_{\text {sup }}^{n} \cap \mathcal{I}=\varnothing$. So, $0 \leq \operatorname{dim} A \leq n-1$. The particular case follows from the inclusion $\mathcal{S}_{r \mathcal{V}_{\text {sup }}^{n}} \subset \mathcal{S}_{r \mathcal{V}_{\text {sup }}^{n}} * \mathcal{I}$. 
(iii) The invariance of the families $\mathcal{I} * \mathcal{S}_{r \mathcal{V}_{\text {sup }}^{n}}$ and $\mathcal{S}_{r \mathcal{V}_{\text {sup }}^{n}} * \mathcal{I}$ under translations of $\mathbb{R}^{n}$ follows from Proposition 5.11, item (ii) and the assumption made on $\mathcal{I}$.

(iv) By Proposition 5.11, we have $\mathcal{S}_{r \mathcal{V}^{n}(Q)} \subset \mathcal{S}_{r \mathcal{V}_{\text {sup }}^{n}}$ for each $Q \in r \mathcal{F}^{n}$. It follows that $\mathcal{S}_{r \mathcal{V}^{n}(Q)} * \mathcal{I} \subseteq \mathcal{S}_{r \mathcal{V}_{\text {sup }}^{n}} * \mathcal{I}$.

Observe that the equality $r \mathcal{V}^{n}(Q) \cap \mathcal{M}^{n}=\varnothing$ for each $Q \in r \mathcal{F}^{n}$ implies that $r \mathcal{V}_{\text {sup }}^{n} \cap \mathcal{M}^{n}=\varnothing$. This observation leads to the following corollary.

Corollary 5.7. The families $\mathcal{M}^{n} * \mathcal{S}_{r \mathcal{V}_{\text {sup }}^{n}}$ and $\mathcal{S}_{r \mathcal{V}_{\text {sup }}^{n}} * \mathcal{M}^{n}$ are semigroups of sets such that $\mathcal{S}_{r \mathcal{V}_{\text {sup }}^{n}} \subset \mathcal{M}^{n} * \mathcal{S}_{r \mathcal{V}_{\text {sup }}^{n}} \subset \mathcal{S}_{r \mathcal{V}_{\text {sup }}^{n}} * \mathcal{M}^{n}$. They are invariant under translations of $\mathbb{R}^{n}$, and consist of sets without the Baire property. In particular, the family $\mathcal{S}_{r} \mathcal{V}_{\text {sup }}^{n}$ consists of sets without the Baire property. Moreover, for each element $A$ of $\mathcal{S}_{r \mathcal{V}_{\text {sup }}^{n}} * \mathcal{M}^{n}$ we have $0 \leq \operatorname{dim} A \leq n-1$.

Proof. Since $\mathcal{M}^{n}$ is invariant under translations of $\mathbb{R}^{n}$ and satisfies the equality $r \mathcal{V}_{\text {sup }}^{n} \cap \mathcal{M}^{n}=\varnothing$, statements in corollary follow from Proposition 5.12. We need to give only one comment.

By remembering that $\mathcal{B}_{p}^{n}=\mathcal{O}^{n} * \mathcal{M}^{n}$, from the equality $\left(\mathcal{S}_{r \mathcal{V}_{\text {sup }}^{n}} * \mathcal{M}^{n}\right) \cap\left(\mathcal{O}^{n} *\right.$ $\left.\mathcal{M}^{n}\right)=\varnothing$, it follows that $\mathcal{S}_{r \mathcal{V}_{\text {sup }}^{n}} * \mathcal{M}^{n} \subset \mathcal{P}\left(\mathbb{R}^{n}\right) \backslash \mathcal{B}_{p}^{n}$. The particular case follows from the inclusion $\mathcal{S}_{r \mathcal{V}_{\text {sup }}^{n}} \subset \mathcal{S}_{r \mathcal{V}_{\text {sup }}^{n}} * \mathcal{M}^{n}$.

Corollary 5.8. Let $\mathcal{I}$ be $\mathcal{I}_{f}, \mathcal{I}_{c}, \mathcal{I}_{c d}$ or $\mathcal{I}_{n}$. Then the families $\mathcal{I} * \mathcal{S}_{r \mathcal{V}_{\text {sup }}^{n}}$ and $\mathcal{S}_{r \mathcal{V}_{\text {sup }}^{n}} * \mathcal{I}$ are semigroups of sets such that $\mathcal{S}_{r \mathcal{V}_{\text {sup }}^{n}} \subset \mathcal{I} * \mathcal{S}_{r \mathcal{V}_{\text {sup }}^{n}} \subset \mathcal{S}_{r \mathcal{V}_{\text {sup }}^{n}} * \mathcal{I}$. They are invariant under translations of $\mathbb{R}^{n}$, and consist of sets without the Baire property having the dimension $\operatorname{dim} \leq n-1$.

Proof. Apply Proposition 5.12.

\subsection{Semigroups of sets in $\mathbb{R}^{n}$ defined by dimension}

Let $Q=\prod_{j=1}^{n} Q_{j}$, where $Q_{j}=\mathbb{Q}$ for each $j=1,2, \cdots, n$. It is clear that $Q$ is a rectangular subgroup of $\left(\mathbb{R}^{n},+\right)$. It follow from Proposition 3.2 and Proposition 3.3, that the families $\mathcal{S}_{r \mathcal{V}^{n}(Q)} * \mathcal{M}^{n}$ and $\mathcal{M}^{n} * \mathcal{S}_{r \mathcal{V}^{n}(Q)}$ are semigroup of sets invariant under translations of $\mathbb{R}^{n}$ such that $\mathcal{S}_{r \mathcal{V}^{n}(Q)} \subset \mathcal{M}^{n} * \mathcal{S}_{r \mathcal{V}^{n}(Q)} \subset \mathcal{S}_{r \mathcal{V}^{n}(Q)} * \mathcal{M}^{n}$.

The following statement extends variety of semigroups of sets without the Baire property based on the family $\mathcal{S}_{r \mathcal{V}^{n}(Q)}$. 
Theorem 5.1. Let $n>1$. Then, there are two finite families $\left\{\mathcal{L}^{n, k}\right\}_{k=0}^{n-1},\left\{\mathcal{R}^{n, k}\right\}_{k=0}^{n-1}$ of pairwise distinct semigroups of sets invariant under translations of the Euclidean space $\mathbb{R}^{n}$ such that:

(a) For each $0 \leq k \leq n-2$ we have $\mathcal{L}^{n, k} \subset \mathcal{L}^{n, k+1}$ and $\mathcal{R}^{n, k} \subset \mathcal{R}^{n, k+1}$.

(b) For each $L \in \mathcal{L}^{n, k}$ and $R \in \mathcal{R}^{n, k}$ we have $\operatorname{dim} L \leq k$ and $\operatorname{dim} R \leq k$ and there are $L_{0} \in \mathcal{L}^{n, k}$ and $R_{0} \in \mathcal{R}^{n, k}$ such that $\operatorname{dim} L_{0}=\operatorname{dim} R_{0}=k$ where $0 \leq k \leq n-1$.

(c) For each $0 \leq k \leq n-1$ we have $\mathcal{L}^{n, k} \subset \mathcal{R}^{n, k}$ but $\mathcal{R}^{n, k-1}$ does not contain $\mathcal{L}^{n, k}$.

(d) $\mathcal{R}^{n, n-1} \subset \mathcal{S}_{r \mathcal{V}^{n}(Q)} * \mathcal{M}^{n}$ but $\mathcal{R}^{n, n-1} \neq \mathcal{S}_{r \mathcal{V}^{n}(Q)} * \mathcal{M}^{n}, \mathcal{L}^{n, n-1} \subset \mathcal{M}^{n} * \mathcal{S}_{r \mathcal{V}^{n}(Q)}$ but $\mathcal{L}^{n, n-1} \neq \mathcal{M}^{n} * \mathcal{S}_{r \mathcal{V}^{n}(Q)}$ and $\mathcal{M}^{n} * \mathcal{S}_{r \mathcal{V}^{n}(Q)}$ does not contain $\mathcal{R}^{n, 0}$, $\mathcal{S}_{r \mathcal{V}^{n}(Q)} \subset \mathcal{L}^{n, 0}$ but $\mathcal{S}_{r \mathcal{V}^{n}(Q)} \neq \mathcal{L}^{n, 0}$.

Proof. For each $0 \leq k<n$, let us consider the family $\mathcal{F}_{k}$ of all closed $k$-dimensional subsets of $\mathbb{R}^{n}$. Note that the family $\mathcal{F}_{k}$ is a semigroup of sets and the inclusions $\mathcal{I}_{\mathcal{F}_{k}} \subset \mathcal{I}_{\mathcal{F}_{k+1}}$ holds for each $0 \leq k \leq n-2$. Since every element of $\mathcal{I}_{\mathcal{F}_{n-1}}$ is nowhere dense in the Euclidean space $\mathbb{R}^{n}$, we have $\mathcal{I}_{\mathcal{F}_{n-1}} \subset \mathcal{M}^{n}$.

For each $0 \leq k<n$ put $\mathcal{R}^{n, k}=\mathcal{S}_{r \mathcal{V}^{n}(Q)} * \mathcal{I}_{\mathcal{F}_{k}}$ and $\mathcal{L}^{n, k}=\mathcal{I}_{\mathcal{F}_{k}} * \mathcal{S}_{r \mathcal{V}^{n}(Q)}$. It follows from Proposition 3.2 and Corollary 3.1 that the families $\mathcal{R}^{n, k}, \mathcal{L}^{n, k}$ are semigroups of sets for each $0 \leq k<n$. It is also clear that the families $\mathcal{R}^{n, k}, \mathcal{L}^{n, k}$ consist of sets which are invariant under translations of $\mathbb{R}^{n}$.

(a) From the inclusion $\mathcal{I}_{\mathcal{F}_{k}} \subset \mathcal{I}_{\mathcal{F}_{k+1}}$, we have $\mathcal{R}^{n, k}=\mathcal{S}_{r \mathcal{V}^{n}(Q)} * \mathcal{I}_{\mathcal{F}_{k}} \subset \mathcal{S}_{r \mathcal{V}^{n}(Q)} *$ $\mathcal{I}_{\mathcal{F}_{k+1}}=\mathcal{R}^{n, k+1}$ and $\mathcal{L}^{n, k}=\mathcal{I}_{\mathcal{F}_{k}} * \mathcal{S}_{r \mathcal{V}^{n}(Q)} \subset \mathcal{I}_{\mathcal{F}_{k+1}} * \mathcal{S}_{r \mathcal{V}^{n}(Q)}=\mathcal{L}^{n, k+1}$.

(b) Let $R \in \mathcal{R}^{n, k}$. Then $R=(U \backslash A) \cup B$ where $U \in \mathcal{S}_{r \mathcal{V}^{n}(Q)}$ and $A, B \in \mathcal{I}_{\mathcal{F}_{k}}$. By Proposition 5.8, we have $\operatorname{dim} U=0$. By monotonicity property of dimension, it follows that $\operatorname{dim}(U \backslash A)=0$.

Note that $R \subseteq(U \backslash A) \cup F$ where $F \in \mathcal{F}_{k}$ and $B \subset F$. Since $F \in \mathcal{F}_{k}$ then $\operatorname{dim} F=k$. By Countable Sum Theorem, $\operatorname{dim}(U \backslash A) \cup F \leq k$. By Monotonicity Theorem, we get $\operatorname{dim} R \leq k$. As $\mathcal{L}^{n, k} \subset \mathcal{R}^{n, k}$, it is clear that for each element $L \in \mathcal{L}^{n, k}$, we have $\operatorname{dim} L \leq k$.

Since for each Vitali set $S$ of $\mathbb{R}^{n}$, the union $S \cup I^{k}=\left(I^{k} \backslash S\right) \cup S=\left(S \backslash I^{k}\right) \cup I^{k}$, where $I^{k}$ is any subset of $\mathbb{R}^{n}$ homeomorphic to the $k$-dimensional cube $[0,1]^{k}$, belongs to both families $\mathcal{L}^{n, k}$ and $\mathcal{R}^{n, k}$ and $\operatorname{dim}\left(S \cup I^{k}\right)=k$, one can take $L_{0}$ and $R_{0}$ to be the set $S \cup I^{k}$. 
(c) By Proposition 3.3, we have the inclusions $\mathcal{L}^{n, k}=\mathcal{I}_{\mathcal{F}_{k}} * \mathcal{S}_{r \mathcal{V}^{n}(Q)} \subset \mathcal{S}_{r \nu^{n}(Q)} *$ $\mathcal{I}_{\mathcal{F}_{k}}=\mathcal{R}^{n, k}$. The item (b) implies that $\mathcal{L}^{n, k-1} \neq \mathcal{L}^{n, k}, \mathcal{R}^{n, k-1} \neq \mathcal{R}^{n, k}$ and the family $\mathcal{R}^{n, k-1}$ can not contain the family $\mathcal{L}^{n, k}$.

(d) For each Vitali set $S$ of $\mathbb{R}^{n}$ the difference $S \backslash\{p\}$, where $p \in S$, can not belong to the family $\mathcal{M}^{n} * \mathcal{S}_{r \mathcal{V}^{n}(Q)}$ but it belongs to the family $\mathcal{S}_{r \mathcal{V}^{n}(Q)} * \mathcal{I}_{\mathcal{F}_{0}}=$ $\mathcal{R}^{n, 0} \subset \mathcal{R}^{n, k}, 0 \leq k \leq n-1$. Hence $\mathcal{L}^{n, k} \neq \mathcal{R}^{n, l}$ for each $0 \leq k, l<n-1$ and $\mathcal{M}^{n} * \mathcal{S}_{r \mathcal{V}^{n}(Q)}$ does not contain $\mathcal{R}^{n, 0}$.

By the inclusion $\mathcal{I}_{\mathcal{F}_{n-1}} \subset \mathcal{M}^{n}$, we have $\mathcal{R}^{n, n-1}=\mathcal{S}_{r \mathcal{V}^{n}(Q)} * \mathcal{I}_{\mathcal{F}_{n-1}} \subset \mathcal{S}_{r \mathcal{V}^{n}(Q)} *$ $\mathcal{M}^{n}$. Again by the inclusion $\mathcal{I}_{\mathcal{F}_{n-1}} \subset \mathcal{M}^{n}$, we have $\mathcal{L}^{n, n-1} \subset \mathcal{M}^{n} * \mathcal{S}_{r \mathcal{V}^{n}(Q)}$ and $\mathcal{S}_{r \mathcal{V}^{n}(Q)} \subset \mathcal{L}^{n, 0}$. In order to finish the proof of (d), let us recall that, by Proposition 5.8, for each element $U \in \mathcal{S}_{r \mathcal{V}^{n}(Q)}$ there are elements $U_{1}, \cdots, U_{n} \in$ $\mathcal{S}_{\mathcal{V}(\mathrm{Q})}$ such that $U \subset \prod_{j=1}^{n} U_{j}$. This implies that no element of $\mathcal{S}_{r \mathcal{V}^{n}(Q)}$ can contain a countable subset of $\mathbb{R}^{n}$ consisting of points with rational coordinates.

The set $\mathbb{C}^{n} \cup S=\left(\mathbb{C}^{n} \backslash S\right) \cup S \in \mathcal{L}^{n, 0}$ where $\mathbb{C}$ is the standard Cantor set of $[0,1]$ and $S$ is any Vitali set of $\mathbb{R}^{n}$, is not any element of $\mathcal{S}_{r \mathcal{V}^{n}(Q)}$. So, $\mathcal{S}_{r \mathcal{V}^{n}(Q)} \neq \mathcal{L}^{n, 0}$.

The set $\mathbb{Q}^{n} \cup S=\left(\mathbb{Q}^{n} \backslash S\right) \cup S=\left(S \backslash \mathbb{Q}^{n}\right) \cup \mathbb{Q}^{n}$, where $\mathbb{Q}$ is the set of all rational numbers of $\mathbb{R}$ and $S$ is any Vitali set of $\mathbb{R}^{n}$, belongs to both families $\mathcal{M}^{n} * \mathcal{S}_{r \mathcal{V}^{n}(Q)}$ and $\mathcal{S}_{r \mathcal{V}^{n}(Q)} * \mathcal{M}^{n}$ but it is not an element of $\mathcal{L}^{n, n-1}$ and $\mathcal{R}^{n, n-1}$. Thus $\mathcal{L}^{n, n-1} \neq \mathcal{M}^{n} * \mathcal{S}_{r \mathcal{V}^{n}(Q)}$ and $\mathcal{R}^{n, n-1} \neq \mathcal{S}_{r \mathcal{V}^{n}(Q)} * \mathcal{M}^{n}$. This complete the proof of (d). 



\section{Conclusion}

The main focus of this thesis was to answer the question about the existence of families of sets without the Baire property, invariant under the action of a nontrivial group of homeomorphisms of the real line $\mathbb{R}$, and on which we can define some algebraic structure.

To settle the problem, we have considered semigroups of sets generated by Vitali $Q$-selectors of $\mathbb{R}$, where $Q$ is a dense countable subgroup of reals, and their extensions obtained by using subideals of the ideal of meager subsets of $\mathbb{R}$.

We have shown that the semigroups of sets generated by Vitali $Q$-selectors as well as their extensions consist of sets without the Baire property and they are invariant under translations of $\mathbb{R}$.

We have observed that in the family of all generated semigroups of sets, where $Q$ is varied, there is no element which contains all others. From here, we constructed a supersemigroup of sets based on all Vitali selectors of $\mathbb{R}$. We have shown that the defined supersemigroup of sets as well as its extensions obtained by using subideals of the ideal of meager subsets of $\mathbb{R}$, are also invariant under translations of $\mathbb{R}$ and consist of sets without the Baire property.

The results from the real line $\mathbb{R}$ were generalized to the finite dimensional Euclidean spaces $\mathbb{R}^{n}, n \geq 2$. Besides the ordinary Vitali selectors of $\mathbb{R}^{n}, n \geq 2$, the rectangular Vitali selectors of $\mathbb{R}^{n}, n \geq 2$ were also considered. In both cases, we have shown that the generated semigroups of sets and their extensions, do 
not possess the Baire property and they are invariant under translations of $\mathbb{R}^{n}$. In this thesis, we have pointed out some questions which still open and where future research should focus on. 


\section{Bibliography}

[1] J.C. Oxtoby, Measure and Category, Springer, 1971.

[2] G. Vitali, "Sul problema della misura dei gruppi di punti di una retta", Bologna, Italy, 1905.

[3] A.B. Kharazishvili, Nonmeasurable Sets and Functions, vol. 195, Elsevier, 2004.

[4] V.A. Chatyrko and V. Nyagahakwa, "On the families of sets without the Baire property generated by the Vitali sets", P-Adic Numbers, Ultrametric Analysis, and Applications, vol. 3, no. 2, pp. 100-107, 2011.

[5] V.A. Chatyrko, "On Vitali sets and their unions", Matematichki Vesnik, vol. 63 , no. 2, pp. 87-92.

[6] M. Aigner, V. A. Chatyrko and V. Nyagahakwa, "The algebra of semigroups of sets".

[7] S.J. Taylor, Introduction to Measure and Integration, CUP Archive, 1973.

[8] A.N. Kolmogorov and S.V. Fomin, Introductory Real Analysis, Courier Dover Publications, 1975.

[9] A. Friedman, Foundations of Modern Analysis, Courier Dover Publications, 1970. 
[10] S. Jitender, "Subgroups of the additive group of real line", Amritsar-Punjab, India, 2013.

[11] T. Jech, Set Theory, The Third Millennium Edition, Revised and Expanded, Springer-Verlag Berlin Heidelberg, 2003.

[12] N. Bourbaki, Elements of Mathematics. General Topology, Pt. 2, Hermann, Addison-Wesley, Paris, Reading, MA, 1974.

[13] R. Engelking, Dimension Theory, North-Holland Publishing Company Amsterdam, 1978.

[14] J. Yeh, Real analysis: Theory of Measure and Integration, World Scientific Hackensack, 2006.

[15] A.B. Kharazishvili, "Measurability properties of Vitali sets", The American Mathematical Monthly, vol. 118, no. 8, pp. 693-703, 2011.

[16] M. Capinski and E. Kopp, Measure, Integral and Probability, Springer, 2004. 